\title{
Bidirectional projection measure of linguistic neutrosophic numbers and their application to multi-criteria group decision making
}

\author{
Peide Liu*, Xinli You \\ School of Management Science and Engineering, Shandong University of Finance and Economics, Jinan, Shandong 250014, China
}

\section{A R T I C L E I N F O}

\section{Keywords:}

Linguistic neutrosophic numbers

Bidirectional projection measure

Distance measure

SHAPLEY weight

MCGDM

\begin{abstract}
A B S T R A C T
Linguistic neutrosophic numbers (LNNs) are an effective tool in describing the incomplete and indeterminate evaluation information by using three linguistic variables (LVs) to denote the truth-degree (TD), indeterminacydegree (ID), and falsity-degree (FD), and the bidirectional projection measure has some advantages in dealing with multi-criteria group decision making (MCGDM) problems because it can consider both the distance and the included angle, but more importantly, it considers the bidirectional projection between each alternative and the ideal solution. In this paper, we define a new distance measure between two linguistic neutrosophic sets (LNSs), and build a model based on the maximum deviation to obtain fuzzy measure, further, we develop the bidirectional projection-based MCGDM method with LNNs in which a weight model based on fuzzy measure is proposed where the weights of evaluation criteria is partial unknown and the interactions among criteria are considered. Finally, we use some examples to verify the effectiveness of the proposed approach and demonstrate its advantages by comparing with some existing methods.
\end{abstract}

\section{Introduction}

In the multi-criteria decision-making (MCDM) or MCGDM problems, the evaluation information is often fuzzy and imprecise due to the limitation of human thought and uncertainty of decision environment. There are numerous researches on fuzzy set (FS) (Zadeh, 1965) or its extensions which are used to properly express ambiguous evaluation information in various fields (Chen, Chin, Li, \& Yang, 2016; Dong, Li, \& Herrera, 2016; Estrella, Espinilla, Herrera, \& Martínez, 2014; MorenteMolinera, Kou, González-Crespo, Corchado, \& Herrera-Viedma, 2017; Peng, Wang, Zhang, \& Chen, 2014; Zhang, Xu, \& Liao, 2017; Zhou \& Xu, 2017). Based on the FS, Smarandache (1999) firstly presented neutrosophic sets (NSs) to describe indeterminate and inconsistent information, in which each element of the universe consists of TD, ID, and FD. In order to simplify the NS and apply it to various fields such as commercial, science, engineering and medicine, Wang, Smarandache, Zhang, and Sunderraman (2010) proposed the concept of single-valued NSs (SVNSs), which are an extension of NSs. Due to their advantages of describing indeterminacy in decision processes, SVNSs have been widely applied to help decision makers (DMs) to make rational and feasible decision. For instance, Liu and Wang (2014) proposed a singlevalued neutrosophic normalized weighted Bonferroni mean (BM) operator for solving practical problems. Peng et al. (2014) extended the
ELECTRE approach to SVNSs. Ye (2014) developed a cross-entropy measurement of SVNSs for MCDM.

In general, for qualitative environment, decision information can be assessed with linguistic terms (LTs) rather than real numbers or fuzzy numbers because of the imprecision of human judgement and the uncertainty of decision environment. In this case, Zadeh (1975) proposed the definition of LVs in 1975. Then, many fuzzy linguistic approaches have been developed and applied to solve problems. Cordon and Herrera (2000) proposed a novel methodology to improve the accuracy of linguistic model. Herrera and Martinez (2000) developed a computational technique to avoid the loss of information in the process of computing with words. As we can see, these methods based on the LVs reflect only the truth/membership degree by default, while the ID and FD cannot be expressed. It is insufficient to accommodate incomplete, indeterminate and inconsistent information in actual decision-making process. In order to overcome this limitation, Ye (2015) presented the single-valued neutrosophic linguistic sets (SVNLSs), which described the TD, ID, and FD of the each element in universe by LVs.

Obviously, the SVNLSs can provide more comprehensive information than LVs, but there is only a single LV in SVNLSs, and the TD, ID and FD are real numbers. In order to overcome the insufficiency of SVNLSs, Fang and Ye (2017) gave the concept of LNN by means of LVs and SVNNs; which is characterized by expressing the TD, ID and FD

\footnotetext{
* Corresponding author.

E-mail address: Peide.liu@gmail.com (P. Liu).
} 
with three LVs rather than exact values. Further, Fan, Ye, Hu, and Fan (2017) developed a LNN normalized weighted BM (LNNNWBM) operator and a LNN normalized weighted geometric BM (LNNNWGBM) operator for group decision. Liang, Zhao, and Wu (2017) proposed a TOPSIS model with LNNs in mining project investment. Shi and Ye (2017) developed a cosine similarity measure under LNNs. In addition, some extended forms of LNNs were proposed to describe the different complex information, such as linguistic neutrosophic cubic number (LNCN) (Ye, 2017a); linguistic cubic variable (LCV) (Ye, 2018b); linguistic neutrosophic uncertain number (LNUN) (Cui, Ye, \& Shi, 2018); hesitant neutrosophic linguistic numbers (HNLNs) (Ye, 2018a), and so on.

The ranking methods of MCGDM or MCDM have recently attracted more and more attentions in different fields. A series of well-known techniques have been built to solve MCDM problems under various fuzzy environments, such as projection model (Ye, 2017d); VIKOR (Ren, Xu, \& Wang, 2017), TOPSIS (Lourenzutti \& Krohling, 2016); AHP (Blagojevic, Srdjevic, Srdjevic, \& Zoranovic, 2016); ELECTRE (Peng, Wang, Wang, Yang, \& Chen, 2015), TODIM (Zhang \& Xu, 2014); and other approaches (Liu \& Guan, 2009; Wei, 2010). Among them, projection measure has its advantage that it can consider both the distance and the included angle between evaluated alternatives. However, the projection measure has its flaws in some cases (see the example in Section 2.5) due to just considering the single directional projection magnitude between the evaluated alternatives and not standardizing the measurement values within [0,1]. In this case, Ye (2017b, 2017c) presented the bidirectional projection measure for the SVNNs and neutrosophic numbers respectively to overcome this shortcoming.

But so far, there is little study on the MCGDM problems based on the LNNs. Based on above analysis, when we select the best alternative(s) under the environment of LNNs, there are two situations need to considered as follows: (1) In practical situations, DMs usually allocate criteria weights from their own preference or judgment, which maybe cause deviation due to the complex decision environment, limited specific knowledge of DMs and so on. What's more, there usually existing interrelationships between criteria. To relieve these impacts and consider the interactions among criteria, we can utilize the maximum deviation method (Wei, 2008) to get the fuzzy measure (Simon, 1971) and determine objective weights of criteria by Shapley weight measure (Shapley, 1953). (2) The bidirectional projection method has the superiority in considering not only the distance and included angle but also the bidirectional projection between each alternative and the ideal solution. Furthermore, the value of bidirectional projection measure is normalized within $[0,1]$ to ensure the rationality and reliability of the final result. Therefore, it is more suitable and valid for the bidirectional projection method to handle MCGDM problems.

As a result, we will propose a new and reasonable MCGDM method this paper, and the main innovations are shown as follows:

(1) Define a new distance measure between two LNSs and prove its relevant properties;

(2) Build a model based on the maximum deviation to obtain fuzzy density, then use Shapley weight to calculate the weight vector of evaluation criteria;

(3) Put forward to the bidirectional projection measure with LNNs. Further, establish the procedure of decision making by the proposed method;

(4) Compare with other existing methods, and illustrate the feasibility and superiority of our proposed methods.

To achieve these goals, the rest of this paper is organized as follows. Section 2 describes preliminaries of LT set, SVNSs, LNNs, the fuzzy measure and the projection and bidirectional projection measures. Section 3 defines the distance of LNSs and builds the weight model based on the fuzzy measure. Section 4 develops a novel MCGDM method based on the bidirectional projection measure with LNNs.
Section 5 describes a numerical example and makes a comparison between the proposed method with those presented approaches in Fang and Ye (2017), Liang et al. (2017), Tu et al. (2018) and Ye (2017b). Section 6 concludes this paper.

\section{Preliminaries}

\subsection{Linguistic term set and linguistic scale function}

A LT set (LTS) $S_{t}=\left\{S_{i} \mid i=0,1,2, \cdots, 2 t\right\}$ is usually a finite and totally ordered discrete set, where $s_{i}$ denotes a LT and $t$ is a positive integer. Meanwhile, $S_{t}$ satisfies the following characteristics (Zadeh, 1975):

(1) The LTS $S_{t}$ is ordered: $s_{i}>s_{j}$ if and only if $i>j$;

(2) A negation operator is defined as: $n e g\left(s_{i}\right)=s_{2 t-i}$.

LTs are usually converted into numerical values by linguistic scale function (LSF), to reduce the loss of information in the integration process.

Definition 1. (Peng \& Wang, 2016). Let $S_{t}=\left\{S_{i} \mid i=1,2, \cdots, 2 t\right\}$ be a discrete LTS, $s_{i}$ be a LT. If $\theta_{i}$ is a numerical value, then the LSF is a mapping from $s_{i}$ to $\theta_{i}(i=0,1, \cdots, 2 t)$, and it can be defined as follows:

$f: s_{i} \rightarrow \theta_{i}(i=0,1, \cdots, 2 t)$

where $f$ is a monotone increasing function, $\theta_{i} \in[0,1]$.

Now, we introduce two kinds of LSFs (Peng \& Wang, 2016):

1. $f_{1}\left(s_{i}\right)=\theta_{i}=\frac{i}{2 t}(i=0,1, \cdots, 2 t)$

This function is defined on the basis of the subscript function and it can evenly distribute the semantic value of the linguistic information.

2. $f_{2}\left(s_{i}\right)=\theta_{i}=\left\{\begin{array}{c}\frac{a^{t}-a^{t-i}}{2 a^{t}-2}(i=0,1, \cdots, t) \\ \frac{a^{t}+a^{i-t}-2}{2 a^{t}-2}(i=t, t+1, \cdots, 2 t)\end{array}\right.$

where $a$ is a parameter which can be determined by experiment. This function has a property that the absolute deviation between adjacent LTs increases from the middle of the given LT to both sides.

\subsection{SVNSS}

Definition 2. (Smarandache, 1999). Let $X$ be a fixed set with the element in $X$ marked as $x$, and $a$ NS $B$ in $X$ can be defined as:

$B=\left\{\left\langle x, T_{B}(x), I_{B}(x), F_{B}(x)\right\rangle \mid x \in X\right\}$

where $T_{B}(x), I_{B}(x)$ and $F_{B}(x)$ denotes the TD, ID and FD of the element $x \in X$ to the set $B$ respectively. For each $\mathrm{x}$ in $X$, it satisfies $\left.T_{B}(x), I_{B}(x), F_{B}(x) \in\right] 0^{-}, 1^{+}\left[\right.$, and $0^{-} \leqslant T_{B}(x)+I_{B}(x)+F_{B}(x) \leqslant 3^{+}$.

Definition 3. (Wang et al., 2010). Let $X$ be a fixed set with the element in $X$ marked as $x$. A SVNS $B$ in $X$ is defined as: $B=\left\{\left\langle x, T_{B}(x), I_{B}(x), F_{B}(x)\right\rangle \mid x \in X\right\}$, where $T_{B}(x), I_{B}(x)$ and $F_{B}(x)$ denotes the TD, ID and FD of the element $x \in X$ to the set $B$ respectively. For each $x$ in $X$, we have $T_{B}(x), I_{B}(x), F_{B}(x) \in[0,1]$, and $0 \leqslant T_{B}(x)+I_{B}(x)+F_{B}(x) \leqslant 3$.

For simplicity, we use $x=(T, I, F)$ to represent an element $x$ in SVNS, and the element $x$ is called a single-valued neutrosophic number (SVNN).

\section{3. $L N N S$}

Definition 4. (Fang \& Ye, 2017). Let $X$ be a fixed set and $G=\left(g_{0}, g_{1}, \cdots, g_{2 t}\right)$ be a LTS. A LNS $H$ in $X$ is composed by a TD $\alpha_{H(x)}$, an ID $\beta_{H(x)}$ and a FD $\gamma_{H(x)}$, where $\alpha_{H(x)}, \beta_{H(x)}, \gamma_{H(x)} \in G$, and $\forall x \in X$, $\eta=\left(g_{\alpha_{H}(x)}, g_{\beta_{H}(x)}, g_{\gamma_{H}(x)}\right) \in H$ is called a LNN of $H$.As a convenience, 
$\Gamma_{[0,2 t]}$ can be used to present the set of all LNNs.

Definition 5. (Fang \& Ye, 2017). Let $\eta=\left(g_{\alpha}, g_{\beta}, g_{\gamma}\right), \eta_{1}=\left(g_{\alpha_{1}}, g_{\beta_{1}}, g_{\gamma_{1}}\right), \eta_{2}=\left(g_{\alpha_{2}}, g_{\beta_{2}}, g_{\gamma_{2}}\right) \in[[0,2 t], \quad \lambda>0$, then the operations of LNNs are shown as follows:

$\eta_{1} \oplus \eta_{2}=\left(g_{\alpha_{1}}, g_{\beta_{1}}, g_{\gamma_{1}}\right) \oplus\left(g_{\alpha_{2}}, g_{\beta_{2}}, g_{\gamma_{2}}\right)=\left(g_{\alpha_{1}+\alpha_{2}-\frac{\alpha_{1} \alpha_{2}}{2 t}}, g_{\frac{\beta_{1} \beta_{2}}{2 t}}, g_{\frac{\gamma_{1} \gamma_{2}}{2 t}}\right)$

$\eta_{1} \otimes \eta_{2}=\left(g_{\alpha_{1}}, g_{\beta_{1}}, g_{\gamma_{1}}\right) \otimes\left(g_{\alpha_{2}}, g_{\beta_{2}}, g_{\gamma_{2}}\right)$

$$
=\left(g_{\frac{\alpha_{1} \alpha_{2}}{2 t}}, g_{\beta_{1}+\beta_{2}-\frac{\beta_{1} \beta_{2}}{2 t}}, g_{\gamma_{1}+\gamma_{2}-\frac{\gamma_{1} \gamma_{2}}{2 t}}\right)
$$

$\lambda \eta=\lambda\left(g_{\alpha}, g_{\beta}, g_{\gamma}\right)=\left(g_{2 t-2 t\left(1-\frac{\alpha}{2 t}\right)^{\lambda}}, g_{\left.2 t\left(\frac{\beta}{2 t}\right)^{\lambda}, g_{2 t}\left(\frac{\gamma}{2 t}\right)^{\lambda}\right)}\right)$

$\eta^{\lambda}=\left(g_{\alpha}, g_{\beta}, g_{\gamma}\right)^{\lambda}=\left(g_{2 t\left(\frac{\alpha}{2 t}\right)^{\lambda}}, g_{2 t-2 t\left(1-\frac{\beta}{2 t}\right)^{\lambda}}, g_{2 t-2 t\left(1-\frac{\gamma}{2 t}\right)^{\lambda}}\right)$

Definition 6. (Fang \& Ye, 2017). Let $\eta=\left(g_{\alpha}, g_{\beta}, g_{\gamma}\right) \in \Gamma_{[0,2 t]}$, we can define the score function $\varphi(\eta)$ and the accuracy function $\sigma(\eta)$ of the LNN $\eta$ as follows:

$\varphi(\eta)=\frac{4 t+\alpha-\beta-\gamma}{6 t}$

$\sigma(\eta)=\frac{\alpha-\gamma}{2 t}$

Definition 7. (Fang \& Ye, 2017). Let $\eta_{1}=\left(g_{\alpha_{1}}, g_{\beta_{1}}, g_{\gamma_{1}}\right), \eta_{2}=\left(g_{\alpha_{2}}, g_{\beta_{2}}, g_{\gamma_{2}}\right) \in \Gamma_{[0,2 t]}$, then

(1) If $\varphi\left(\eta_{1}\right)<\varphi\left(\eta_{2}\right)$, then $\eta_{1}<\eta_{2}$;

(2) If $\varphi\left(\eta_{1}\right)=\varphi\left(\eta_{2}\right)$

(a) and $\sigma\left(\eta_{1}\right)<\sigma\left(\eta_{2}\right)$, then $\eta_{1}<\eta_{2}$;

(b) and $\sigma\left(\eta_{1}\right)=\sigma\left(\eta_{2}\right)$, then $\eta_{1}=\eta_{2}$.

Definition 8. (Fang \& Ye, 2017). Let $\eta_{i}=\left(g_{\alpha_{i}}, g_{\beta_{i}}, g_{\gamma_{i}}\right)(i=1,2, \cdots, n)$ be a collection of LNNs, the linguistic neutrosophic weighted arithmetic averaging (LNWAA) operator is defined as

$$
\begin{aligned}
\operatorname{LNWAA}\left(\eta_{1}, \eta_{2}, \ldots, \eta_{n}\right) & =\sum_{i=1}^{n} \omega_{i} \eta_{i} \\
& =\left(g_{2 t-2 t} \prod_{i=1}^{n}\left(1-\frac{\alpha_{i}}{2 t}\right)^{\omega_{i},} g_{2 t} \prod_{i=1}^{n}\left(\frac{\beta_{i}}{2 t}\right)^{\omega_{i}}, g_{2 t} \prod_{i=1}^{n}\left(\frac{\gamma_{i}}{2 t}\right)^{\omega_{i}}\right)
\end{aligned}
$$

where $\omega=\left(\omega_{1}, \omega_{2}, \ldots, \omega_{n}\right)^{T}$ is the weight vector of $\left(\eta_{1}, \eta_{2}, \cdots, \eta_{n}\right)$, $\omega_{i} \in[0,1]$ and $\sum_{i=1}^{k} \omega_{i}=1$.

Definition 9. (Fang \& Ye, 2017). Let $\eta_{i}=\left(g_{\alpha_{i}}, g_{\beta_{i}}, g_{\gamma_{i}}\right)(i=1,2, \cdots, n)$ be a collection of LNNs, the linguistic neutrosophic weight geometric averaging (LNWGA) operator is defined as

$$
\begin{aligned}
\operatorname{LNWGA}\left(\eta_{1}, \eta_{2}, \ldots ., \eta_{n}\right)= & \sum_{i=1}^{n} \eta_{i}^{\omega_{i}} \\
= & \left(g_{2 t} \prod_{i=1}^{n}\left(\frac{\alpha_{i}}{2 t}\right)^{\omega_{i}}, g_{2 t-2 t} \prod_{i=1}^{n}\left(1-\frac{\beta_{i}}{2 t}\right)^{\omega_{i}}\right. \\
& \left., g_{2 t-2 t} \prod_{i=1}^{n}\left(1-\frac{\gamma_{i}}{2 t}\right)^{\omega_{i}}\right)
\end{aligned}
$$

where $\omega=\left(\omega_{1}, \omega_{2}, \ldots, \omega_{n}\right)^{T}$ is the weight vector of $\left(\eta_{1}, \eta_{2}, \cdots, \eta_{n}\right)$, $\omega_{i} \in[0,1]$ and $\sum_{i=1}^{k} \omega_{i}=1$.

\subsection{The fuzzy measure}

Fuzzy measure (Simon, 1971) is an effective tool that can be used to measure the interaction among evaluation criteria, which is described as follows:

Definition 10. (Simon, 1971). Let $X=\left\{x_{1}, x_{2}, \cdots, x_{n}\right\}$ be a fixed set. A fuzzy measure on $X$ is a set function $\mu: \psi(X) \rightarrow[0,1]$ which satisfies the following properties:

(1) Boundary: $\mu(\phi)=0, \mu(X)=1$,

(2) Monotonicity: If $M, N \in \psi(X)$ and $M \subseteq N$, then $\mu(M)<\mu(N)$,

Definition 11. (Sugeno, 1974). Let $X=\left\{x_{1}, x_{2}, \cdots, x_{n}\right\}$ be a fixed set. A fuzzy measure $\rho$ on $X$ is called $\lambda$-fuzzy measure when it meets the following criteria: $\rho(M \cup N)=\rho(M)+\rho(N)+\lambda \rho(M) \rho(N), \quad \lambda \in$ $[-1, \infty), \forall M, N \in P(X)$ and $M \cap N=\phi$, where $P(X)$ is the power set of $X$.

Meanwhile, the $\lambda$-fuzzy measure of $A$ can be obtained if $A \in P(X)$ as follows:

$\rho(A)=\left\{\begin{array}{c}\frac{1}{\lambda}\left(\prod_{i \in A}[1+\lambda \rho(i)]-1\right) \lambda \neq 0 \\ \sum_{i \in A} \rho(i) \lambda=0\end{array}\right.$

where $\rho(i)$ is usually called the fuzzy density because it's a $\lambda$-fuzzy measure for a subset with a single elementi. Since $\rho(X)=1$, then the value of $\lambda$ can be determined by solving $\lambda+1=\prod_{i=1}^{n}[1+\lambda \rho(i)]$.

Many researches based on fuzzy measures have been developed, such as Choquet integral (Grabisc, Nguyen, \& Walker, 1995) and Shapley weight (Shapley, 1953). The Shapley weight indicates the coefficient of importance. It can not only measure the importance for criteria but also reflects the interactive characteristics among them; which can be expressed by

$\varphi_{i}(\rho, X)=\sum_{T \subseteq X / x_{i}} \frac{(n-t-1) ! t !}{n !}\left[\rho\left(T \cup x_{i}\right)-\rho(T)\right]$

where $n, t$ denotes the cardinality of set $X, T$, respectively.

\subsection{Projection and bidirectional projection measures}

Definition 12. $(X u, 2005)$. Let $\chi=\left(\chi_{1}, \chi_{2}, \cdots, \chi_{m}\right)$ and $\lambda=\left(\lambda_{1}, \lambda_{2}, \cdots, \lambda_{m}\right)$ be two vectors, then

$\cos (\chi, \lambda)=\frac{\sum_{j=1}^{m} \chi_{j} \lambda_{j}}{\|\chi\| \cdot\|\lambda\|}=\frac{\sum_{j=1}^{m} \chi_{j} \lambda_{j}}{\sqrt{\sum_{j=1}^{m} \chi_{j}^{2}} \cdot \sqrt{\sum_{j=1}^{m} \lambda_{j}^{2}}}$

is called the cosine of the included angle between $\chi$ and $\lambda$, where $\sqrt{\sum_{j=1}^{m} \chi_{j}^{2}}$ and $\sqrt{\sum_{j=1}^{m} \lambda_{j}^{2}}$ are the modules of $\chi$ and $\lambda$ respectively, denoted by $\|\chi\|$ and $\|\lambda\|$.

Obviously, $0<\cos (\chi, \lambda) \leqslant 1$, and the greater the cosine value of the angle between $\chi$ and $\lambda$, the closer $\chi$ and $\lambda$.

Definition 13. $(X u, 2005)$. Let $\chi=\left(\chi_{1}, \chi_{2}, \cdots, \chi_{m}\right)$ and $\lambda=\left(\lambda_{1}, \lambda_{2}, \cdots, \lambda_{m}\right)$ be two vectors, then

$P_{r o j \lambda}(\chi)=\|\chi\| \cos (\chi, \lambda)=\sqrt{\sum_{j=1}^{m} \chi_{j}^{2}} \cdot \frac{\sum_{j=1}^{m} \chi_{j} \lambda_{j}}{\sqrt{\sum_{j=1}^{m} \chi_{j}^{2}} \cdot \sqrt{\sum_{j=1}^{m} \lambda_{j}^{2}}}=\frac{\sum_{j=1}^{m} \chi_{j} \lambda_{j}}{\sqrt{\sum_{j=1}^{m} \lambda_{j}^{2}}}$

is called the projection of the vector $\chi$ on the vector $\lambda . P_{\text {roj } \lambda}(\chi)$.

The projection measure $P_{r o j} \lambda(\chi)$ needs to consider both the distance and the included angle between $\chi$ and $\lambda$. In general, the larger the value of $P_{r o j} \lambda(\chi)$ is, the closer $\chi$ is to $\lambda$.

However, there are some unreasonable case in the projection measurement. 
Example 1.. let $\chi=\lambda=\left(\chi_{1}, \chi_{2}, \cdots, \chi_{m}\right)$ and $\varphi=\left(2 \chi_{1}, 2 \chi_{2}, \cdots, 2 \chi_{m}\right)$, then $P_{r o j \lambda}(\chi)=\|\chi\|$ and $P_{r o j} \lambda(\varphi)=2\|\chi\|$.

Clearly, $P_{r o j} \lambda(\varphi)$ is larger than $P_{r o j} \lambda(\chi)$. But we can see the fact is, $\chi$ is closer to $\lambda$ than $\varphi$ because $\chi=\lambda$. Hence, sometimes the projection is not able to depict the closeness between $\chi$ to $\lambda$ accurately.

To overcome the shortcoming mentioned above in general projection, Ye (2017c) proposed the bidirectional projection model as following.

Definition 14. $(Y e, 2017 c)$. Let $x=\left(\left[x_{1}^{l}, x_{1}^{u}\right],\left[x_{2}^{l}, x_{2}^{u}\right], \cdots,\left[x_{m}^{l}, x_{m}^{u}\right]\right)$ and $y=\left(\left[y_{1}^{l}, y_{1}^{u}\right],\left[y_{2}^{l}, y_{2}^{u}\right], \cdots,\left[y_{m}^{l}, y_{m}^{u}\right]\right)$ be two interval vectors, then

$B P_{r o j}(x, y)=\frac{1}{1+\left|\frac{x \cdot y}{\|x\|}-\frac{x \cdot y}{\|y\|}\right|}=\frac{\|x\|\|y\|}{\|x\|\|y\|+|\|x\|-\|y\|| x \cdot y}$

is called the bidirectional projection between $x$ and $y$, where $\|x\|=\sqrt{\sum_{i=1}^{m}\left(\left(x_{i}^{l}\right)^{2}+\left(x_{i}^{u}\right)^{2}\right)}$ and $\|y\|=\sqrt{\sum_{i=1}^{m}\left(\left(y_{i}^{l}\right)^{2}+\left(y_{i}^{u}\right)^{2}\right)}$ are the modules of $x$ and $y$ respectively.

The bidirectional projection model can consider both the distance and the included angle between $x$ and $y$, and it considers the bidirectional projection between $x$ and $y$. In addition, the value of bidirectional projection measure is bounded within $[0,1]$, the closer the value of $B P_{r o j}(x, y)$ is to 1 , the closer $x$ is to $y$. When, and only when $x=y$, then $B P_{r o j}(x, y)=1$.

\section{Weight determination model based on fuzzy measure}

In this section, we firstly redefine the distance of LNSs. Then, an extended weight determination model is provided by combining the maximum deviation method and fuzzy measure.

\subsection{The distance between LNSS}

In Ref. Liang et al. (2017); Liang et al. gave the axioms of distance measure between any two LNSs.

Definition 15 (Liang et al., 2017.). Let $G=\left(g_{0}, g_{1}, \cdots, g_{2 t}\right)$, for any $A, B, C \in L N S s(X)$, if $d(A, B)$ satisfies the following properties:

(1) $0 \leqslant d(A, B) \leqslant 1$;

(2) $d(A, B)=d(B, A)$;

(3) $d(A, B)=0$ if and only if $A=B$;

(4) $d(A, C) \leqslant d(A, B)+d(B, C)$.

then $d(A, B)$ is a distance measure between LNSs $A$ and $B$.

Definition 16 (Liang et al., 2017.). Let $X$ be the universe of discourse, where $X=\left\{x_{1}, x_{2}, \cdots, x_{n}\right\}$, and let $A$ and $B$ be two LNSs in the $X$, where $\quad A=\left\{\left\langle x_{i}, g_{\alpha_{A}\left(x_{i}\right)}, g_{\beta_{A}\left(x_{i}\right)}, g_{\gamma_{A}\left(x_{i}\right)}\right\rangle \mid x_{i} \in X\right\} \quad$ and $B=$ $\left\{\left\langle x_{i}, g_{\alpha_{B}\left(x_{i}\right)}, g_{\beta_{B}\left(x_{i}\right)}, g_{\gamma_{B}\left(x_{i}\right)}\right\rangle \mid x_{i} \in X\right\}$. Then, the distance between $A$ and $B$ is defined as follows:

$$
\begin{aligned}
d_{G}(A, B)= & \left(\frac { 1 } { 3 n } \sum _ { i = 1 } ^ { n } \left(\left|\frac{\alpha_{A}\left(x_{i}\right)}{2 t}-\frac{\alpha_{B}\left(x_{i}\right)}{2 t}\right|^{\lambda}+\left|\frac{\beta_{A}\left(x_{i}\right)}{2 t}-\frac{\beta_{B}\left(x_{i}\right)}{2 t}\right|^{\lambda}, \lambda>0\right.\right. \\
& \left.\left.+\left|\frac{\gamma_{A}\left(x_{i}\right)}{2 t}-\frac{\gamma_{B}\left(x_{i}\right)}{2 t}\right|^{\lambda}\right)\right)^{\frac{1}{\lambda}}
\end{aligned}
$$

Obviously, we have some particular cases as follows.

(1) if $\lambda=1$, it is a Hamming distance as follow.

$$
\begin{aligned}
d_{H}(A, B)= & \frac{1}{3 n} \sum_{i=1}^{n}\left(\left|\frac{\alpha_{A}\left(x_{i}\right)}{2 t}-\frac{\alpha_{B}\left(x_{i}\right)}{2 t}\right|+\left|\frac{\beta_{A}\left(x_{i}\right)}{2 t}-\frac{\beta_{B}\left(x_{i}\right)}{2 t}\right|\right. \\
& \left.+\left|\frac{\gamma_{A}\left(x_{i}\right)}{2 t}-\frac{\gamma_{B}\left(x_{i}\right)}{2 t}\right|\right)
\end{aligned}
$$

(2) if $\lambda=2$, it is a Euclidean distance as follow.

$$
\begin{aligned}
& d_{E}(A, B)=\sqrt{\frac{1}{3 n} \sum_{i=1}^{n}\left(\left|\frac{\alpha_{A}\left(x_{i}\right)}{2 t}-\frac{\alpha_{B}\left(x_{i}\right)}{2 t}\right|^{2}+\left|\frac{\beta_{A}\left(x_{i}\right)}{2 t}-\frac{\beta_{B}\left(x_{i}\right)}{2 t}\right|^{2}\right.} \\
& \left(+\left|\frac{\gamma_{A}\left(x_{i}\right)}{2 t}-\frac{\gamma_{B}\left(x_{i}\right)}{2 t}\right|^{2}\right)
\end{aligned}
$$

(3) when $\lambda \rightarrow \infty$, it is a Hausdorff distance as follow.

$$
\begin{aligned}
d_{H d}(A, B)= & \max \left\{\sum _ { i = 1 } ^ { n } \left(\left|\frac{\alpha_{A}\left(x_{i}\right)}{2 t}-\frac{\alpha_{B}\left(x_{i}\right)}{2 t}\right|,\left|\frac{\beta_{A}\left(x_{i}\right)}{2 t}-\frac{\beta_{B}\left(x_{i}\right)}{2 t}\right|, \mid \frac{\gamma_{A}\left(x_{i}\right)}{2 t}\right.\right. \\
& \left.\left.-\frac{\gamma_{B}\left(x_{i}\right)}{2 t} \mid\right)\right\}
\end{aligned}
$$

Although the above distance measures between LNSs satisfies the distance axioms (Definition 15), some unreasonable cases can still be found. For example, we suppose LNNs $\eta_{1}=\left(g_{5}, g_{1}, g_{4}\right), \eta_{2}=\left(g_{4}, g_{3}, g_{4}\right), \eta_{3}=\left(g_{4}, g_{2}, g_{1}\right) \in[0,6]$. In this case, the distance between $\eta_{1}$ and $\eta_{3}$ calculated by Formula (16) is greater than that between $\eta_{2}$ and $\eta_{3}$. It seems to be unreasonable since LNNs $\eta_{1}, \eta_{2}$, and $\eta_{3}$ are ordered as $\eta_{3}>\eta_{1}>\eta_{2}$ on the basis of the comparison method given in Definition 7. The sorted result indicates that the distance between $\eta_{1}$ and $\eta_{3}$ is smaller than that between $\eta_{2}$ and $\eta_{3}$.

As a powerful tool in modeling uncertain information, LNSs have the advantage of both reflecting specific information: TD and FD, and non-specific information: ID. It should be noted that the main goal of distance measure is to measure the difference of information carried by LNSs. Therefore, combining the above equations, we redefine a generalized hybrid distance as follows:

Definition 17.. Let $G=\left(g_{0}, g_{1}, \cdots, g_{2 t}\right)$, for any $A, B, C \in \operatorname{LNSs}(X)$,

$$
\begin{aligned}
& d_{L}(A, B)=\frac{1}{3 n} \sum_{i=1}^{n}\left[\theta_{1}\left(x_{i}\right)+\theta_{2}\left(x_{i}\right)+\theta_{3}\left(x_{i}\right)\right] \\
& \begin{aligned}
\theta_{1}\left(x_{i}\right)= & \frac{1}{2}\left(\left|\frac{\alpha_{A}\left(x_{i}\right)}{2 t}-\frac{\alpha_{B}\left(x_{i}\right)}{2 t}\right|+\left|\frac{\gamma_{A}\left(x_{i}\right)}{2 t}-\frac{\gamma_{B}\left(x_{i}\right)}{2 t}\right|\right) \\
\theta_{2}\left(x_{i}\right) & =\frac{1}{2}\left(\frac{\beta_{A}\left(x_{i}\right)}{2 t}+\frac{\beta_{B}\left(x_{i}\right)}{2 t}\right) \theta_{3}\left(x_{i}\right) \\
= & \max \left\{\left|\frac{\alpha_{A}\left(x_{i}\right)}{2 t}-\frac{\alpha_{B}\left(x_{i}\right)}{2 t}\right|,\left|\frac{\gamma_{A}\left(x_{i}\right)}{2 t}-\frac{\gamma_{B}\left(x_{i}\right)}{2 t}\right|\right\}
\end{aligned}
\end{aligned}
$$

Proof:. (1) According to the Definition 17, $0 \leqslant \frac{\alpha_{A}\left(x_{i}\right)}{2 t}, \frac{\beta_{A}\left(x_{i}\right)}{2 t}, \frac{\gamma_{A}\left(x_{i}\right)}{2 t}, \frac{\alpha_{B}\left(x_{i}\right)}{2 t}, \frac{\beta_{B}\left(x_{i}\right)}{2 t}, \frac{\gamma_{B}\left(x_{i}\right)}{2 t} \leqslant 1$, we can obtain $0 \leqslant\left|\frac{\alpha_{A}\left(x_{i}\right)}{2 t}-\frac{\alpha_{B}\left(x_{i}\right)}{2 t}\right|,\left|\frac{\gamma_{A}\left(x_{i}\right)}{2 t}-\frac{\gamma_{B}\left(x_{i}\right)}{2 t}\right|, \frac{1}{2}\left(\frac{\beta_{A}\left(x_{i}\right)}{2 t}+\frac{\beta_{B}\left(x_{i}\right)}{2 t}\right) \leqslant 1$, then $0 \leqslant \theta_{1}\left(x_{i}\right) \leqslant 1,0 \leqslant \theta_{2}\left(x_{i}\right) \leqslant 1,0 \leqslant \theta_{3}\left(x_{i}\right) \leqslant 1$, so $0 \leqslant d(A, B) \leqslant 1$.

(2) $d_{L}(A, B)$

$$
=\frac{1}{3 n} \sum_{i=1}^{n}\left[\begin{array}{c}
\frac{1}{2}\left(\left|\frac{\alpha_{A}\left(x_{i}\right)}{2 t}-\frac{\alpha_{B}\left(x_{i}\right)}{2 t}\right|+\left|\frac{\gamma_{A}\left(x_{i}\right)}{2 t}-\frac{\gamma_{B}\left(x_{i}\right)}{2 t}\right|\right)+\frac{1}{2}\left(\frac{\beta_{A}\left(x_{i}\right)}{2 t}+\frac{\beta_{B}\left(x_{i}\right)}{2 t}\right) \\
+\max \left(\left|\frac{\alpha_{A}\left(x_{i}\right)}{2 t}-\frac{\alpha_{B}\left(x_{i}\right)}{2 t}\right|,\left|\frac{\gamma_{A}\left(x_{i}\right)}{2 t}-\frac{\gamma_{B}\left(x_{i}\right)}{2 t}\right|\right)
\end{array}\right]
$$

$\frac{1}{3 n} \sum_{i=1}^{n}\left[\begin{array}{c}\frac{1}{2}\left(\left|\frac{\alpha_{B}\left(x_{i}\right)}{2 t}-\frac{\alpha_{A}\left(x_{i}\right)}{2 t}\right|+\left|\frac{\gamma_{B}\left(x_{i}\right)}{2 t}-\frac{\gamma_{A}\left(x_{i}\right)}{2 t}\right|\right)+\frac{1}{2}\left(\frac{\beta_{B}\left(x_{i}\right)}{2 t}+\frac{\beta_{A}\left(x_{i}\right)}{2 t}\right) \\ +\max \left(\left|\frac{\alpha_{B}\left(x_{i}\right)}{2 t}-\frac{\alpha_{A}\left(x_{i}\right)}{2 t}\right|,\left|\frac{\gamma_{B}\left(x_{i}\right)}{2 t}-\frac{\gamma_{A}\left(x_{i}\right)}{2 t}\right|\right)\end{array}\right]=d_{L}$

$(B, A)$

(3) $d_{L}(A, B)=0 \Leftrightarrow \alpha_{A}\left(x_{i}\right)=\alpha_{B}\left(x_{i}\right), \gamma_{A}\left(x_{i}\right)=\gamma_{B}\left(x_{i}\right), \beta_{A}\left(x_{i}\right)$

$$
=\beta_{B}\left(x_{i}\right)=0 \Leftrightarrow A(x)=B(x), \beta_{A}\left(x_{i}\right)=\beta_{B}\left(x_{i}\right)=0
$$


(4) $d_{L}(A, C)$

$=\frac{1}{3 n} \sum_{i=1}^{n}\left[\begin{array}{c}\frac{1}{2}\left(\left|\frac{\alpha_{A}\left(x_{i}\right)}{2 t}-\frac{\alpha_{C}\left(x_{i}\right)}{2 t}\right|+\left|\frac{\gamma_{A}\left(x_{i}\right)}{2 t}-\frac{\gamma_{C}\left(x_{i}\right)}{2 t}\right|\right)+\frac{1}{2}\left(\frac{\beta_{A}\left(x_{i}\right)}{2 t}+\frac{\beta_{C}\left(x_{i}\right)}{2 t}\right) \\ +\max \left(\left|\frac{\alpha_{A}\left(x_{i}\right)}{2 t}-\frac{\alpha_{C}\left(x_{i}\right)}{2 t}\right|,\left|\frac{\gamma_{A}\left(x_{i}\right)}{2 t}-\frac{\gamma_{C}\left(x_{i}\right)}{2 t}\right|\right)\end{array}\right]$

$d_{L}(A, B)$

$=\frac{1}{3 n} \sum_{i=1}^{n}\left[\begin{array}{c}\frac{1}{2}\left(\left|\frac{\alpha_{A}\left(x_{i}\right)}{2 t}-\frac{\alpha_{B}\left(x_{i}\right)}{2 t}\right|+\left|\frac{\gamma_{A}\left(x_{i}\right)}{2 t}-\frac{\gamma_{B}\left(x_{i}\right)}{2 t}\right|\right)+\frac{1}{2}\left(\frac{\beta_{A}\left(x_{i}\right)}{2 t}+\frac{\beta_{B}\left(x_{i}\right)}{2 t}\right) \\ +\max \left(\left|\frac{\alpha_{A}\left(x_{i}\right)}{2 t}-\frac{\alpha_{B}\left(x_{i}\right)}{2 t}\right|,\left|\frac{\gamma_{A}\left(x_{i}\right)}{2 t}-\frac{\gamma_{B}\left(x_{i}\right)}{2 t}\right|\right)\end{array}\right]$

$d_{L}(B, C)$

$$
=\frac{1}{3 n} \sum_{i=1}^{n}\left[\begin{array}{c}
\frac{1}{2}\left(\left|\frac{\alpha_{B}\left(x_{i}\right)}{2 t}-\frac{\alpha_{C}\left(x_{i}\right)}{2 t}\right|+\left|\frac{\gamma_{B}\left(x_{i}\right)}{2 t}-\frac{\gamma_{C}\left(x_{i}\right)}{2 t}\right|\right)+\frac{1}{2}\left(\frac{\beta_{B}\left(x_{i}\right)}{2 t}+\frac{\beta_{C}\left(x_{i}\right)}{2 t}\right) \\
+\max \left(\left|\frac{\alpha_{B}\left(x_{i}\right)}{2 t}-\frac{\alpha_{C}\left(x_{i}\right)}{2 t}\right|,\left|\frac{\gamma_{B}\left(x_{i}\right)}{2 t}-\frac{\gamma_{C}\left(x_{i}\right)}{2 t}\right|\right)
\end{array}\right]
$$

It is obvious that

$$
\begin{aligned}
& \left|\frac{\alpha_{A}\left(x_{i}\right)}{2 t}-\frac{\alpha_{C}\left(x_{i}\right)}{2 t}\right|=\left|\frac{\alpha_{A}\left(x_{i}\right)}{2 t}-\frac{\alpha_{B}\left(x_{i}\right)}{2 t}+\frac{\alpha_{B}\left(x_{i}\right)}{2 t}-\frac{\alpha_{C}\left(x_{i}\right)}{2 t}\right| \leqslant \\
& \left|\frac{\alpha_{A}\left(x_{i}\right)}{2 t}-\frac{\alpha_{B}\left(x_{i}\right)}{2 t}\right|+\left|\frac{\alpha_{B}\left(x_{i}\right)}{2 t}-\frac{\alpha_{C}\left(x_{i}\right)}{2 t}\right| \\
& \left|\frac{\gamma_{A}\left(x_{i}\right)}{2 t}-\frac{\gamma_{C}\left(x_{i}\right)}{2 t}\right|=\left|\frac{\gamma_{A}\left(x_{i}\right)}{2 t}-\frac{\gamma_{B}\left(x_{i}\right)}{2 t}+\frac{\gamma_{B}\left(x_{i}\right)}{2 t}-\frac{\gamma_{C}\left(x_{i}\right)}{2 t}\right| \leqslant \\
& \left|\frac{\gamma_{A}\left(x_{i}\right)}{2 t}-\frac{\gamma_{B}\left(x_{i}\right)}{2 t}\right|+\left|\frac{\gamma_{B}\left(x_{i}\right)}{2 t}-\frac{\gamma_{C}\left(x_{i}\right)}{2 t}\right| \\
& \frac{\beta_{A}\left(x_{i}\right)}{2 t}+\frac{\beta_{C}\left(x_{i}\right)}{2 t} \leqslant \frac{\beta_{A}\left(x_{i}\right)}{2 t}+\frac{\beta_{B}\left(x_{i}\right)}{2 t}+\frac{\beta_{B}\left(x_{i}\right)}{2 t}+\frac{\beta_{C}\left(x_{i}\right)}{2 t}
\end{aligned}
$$

Thus, we have $d_{L}(A, C) \leqslant d_{L}(A, B)+d_{L}(B, C)$.

Example 2.. Let $\eta_{1}=\left(\mathrm{g}_{5}, \mathrm{~g}_{1}, \mathrm{~g}_{4}\right), \eta_{2}=\left(\mathrm{g}_{4}, \mathrm{~g}_{3}, \mathrm{~g}_{4}\right), \eta_{3}=\left(\mathrm{g}_{4}, \mathrm{~g}_{2}, \mathrm{~g}_{1}\right) \in \Gamma_{[0,6]}$. According to the new distance measure in Definition 17, we can obtain

$$
\begin{aligned}
d_{L}\left(\eta_{1}, \eta_{2}\right)= & \frac{1}{3}\left[\frac{1}{2}\left(\left|\frac{5}{6}-\frac{4}{6}\right|+\left|\frac{4}{6}-\frac{4}{6}\right|\right)\right. \\
& \left.+\frac{1}{2}\left(\frac{1}{6}+\frac{3}{6}\right)+\max \left(\left|\frac{5}{6}-\frac{4}{6}\right|,\left|\frac{5}{6}-\frac{4}{6}\right|\right)\right]=\frac{7}{36} \\
d_{L}\left(\eta_{1}, \eta_{3}\right)= & \frac{1}{3}\left[\frac{1}{2}\left(\left|\frac{5}{6}-\frac{4}{6}\right|+\left|\frac{4}{6}-\frac{1}{6}\right|\right)\right. \\
& \left.+\frac{1}{2}\left(\frac{1}{6}+\frac{2}{6}\right)+\max \left(\left|\frac{5}{6}-\frac{4}{6}\right|,\left|\frac{4}{6}-\frac{1}{6}\right|\right)\right]=\frac{13}{36} \\
d_{L}\left(\eta_{2}, \eta_{3}\right)= & \frac{1}{3}\left[\frac{1}{2}\left(\left|\frac{4}{6}-\frac{4}{6}\right|+\left|\frac{4}{6}-\frac{1}{6}\right|\right)\right. \\
& \left.+\frac{1}{2}\left(\frac{3}{6}+\frac{2}{6}\right)+\max \left(\left|\frac{4}{6}-\frac{4}{6}\right|,\left|\frac{4}{6}-\frac{1}{6}\right|\right)\right]=\frac{14}{36}
\end{aligned}
$$

From the results, we can seed $d_{L}\left(\eta_{2}, \eta_{3}\right)>d_{L}\left(\eta_{1}, \eta_{3}\right)$, which is in line with the ordered result $\eta_{3}>\eta_{1}>\eta_{2}$. Therefore, the proposed distance measure is more reasonable.

\subsection{A weight determination model based on fuzzy measure}

Generally, fuzzy measure $\mu(C)$ can be regarded as the subjective importance level of criterion $C$ in MCDM problems. However, the weights of criteria are usually partial known, we use maximum deviation method to get the fuzzy measure $\mu(C)$.

In a MCDM problem with interaction criteria, suppose alternative set is $A=\left(A_{1}, A_{2}, \cdots, A_{m}\right)$, criteria set is $C=\left(C_{1}, C_{2}, \cdots, C_{n}\right)$, then the evaluation values of all alternatives under criteria set $C$ can be expressed by LNNs $r_{i j}=\left(g_{\alpha_{i j}}, g_{\beta_{i j}}, g_{\gamma_{i j}}\right) \quad$ with $\alpha_{i j}, \beta_{i j}, \gamma_{i j} \in[0,2 t]$ $(i=1,2, \ldots, m ; j=1,2, \ldots, n)$. Due to the weights of criteria $w_{C_{j}}$ is partially known, suppose $w_{C_{j}} \in H_{C_{j}}$, where $H_{C_{j}}$ expresses the range of weight for criteria $w_{C_{j}}$ which is described by $H_{C_{j}}=\left[H_{C_{j}}^{-}, H_{C_{j}}^{+}\right]$. Further, we use $H$ to express the ranges of weights for all criteria.

Firstly, we define the deviation degree of $A_{i}(i=1,2, \ldots, m)$ to all alternatives with respect to criteria $C_{j}(j=1,2, \ldots ., n)$ as follows:

$D_{i j}\left(\varphi_{C_{j}}(\mu, C)\right)=\sum_{k=1}^{m} d\left(r_{i j}, r_{k j}\right) \varphi_{C_{j}}(\mu, C)$

where $d\left(r_{i j}, r_{k j}\right)$ is the distance between $r_{i j}$ and $r_{k j}, \varphi_{C_{j}}(\mu, C)$ is the Shapley weight of criterion $C_{j}, \mu$ is the fuzzy measure of $C_{j}(j=1,2, \ldots ., n)$.

Then, the deviation degrees of all alternatives under the criteria $C_{j}(j=1,2, \ldots, n)$ are expressed by

$D_{j}\left(\varphi_{C_{j}}(\mu, C)\right)=\sum_{i=1}^{m} D_{i j}\left(\varphi_{C_{j}}(\mu, C)\right)=\sum_{i=1}^{m} \sum_{k=1}^{m} d\left(r_{i j}, r_{k j}\right) \varphi_{C_{j}}(\mu, C)$

Thus, the total deviation of all alternatives with all criteria is proposed as follows:

$D(\varphi(\mu, C))=\sum_{j=1}^{n} D_{j}\left(\varphi_{C_{j}}(\mu, C)\right)=\sum_{j=1}^{n} \sum_{i=1}^{m} \sum_{k=1}^{m} d\left(a_{i j}, a_{k j}\right) \varphi_{C_{j}}(\mu, C)$

So we can construct the maximum deviation model and obtain the fuzzy density of each criteria and criteria set:

$$
\begin{gathered}
\max D(\varphi(\mu, C))=\sum_{j=1}^{n} \sum_{i=1}^{m} \sum_{k=1}^{m} d\left(a_{i j}, a_{k j}\right) \varphi_{C_{j}}(\mu, C) \\
\text { s.t. }\left\{\begin{array}{c}
\mu(\phi)=0, \mu(C)=1 \\
\mu(S) \leqslant \mu(T), \forall S, T \subseteq C, S \subseteq T \\
\mu\left(C_{j}\right) \in H_{C_{j}}
\end{array}\right.
\end{gathered}
$$

Finally, the $\lambda$-fuzzy measure of criteria can be obtained by formula (12), and the Shapley weight of each criterion can be calculated by means of formula (13).

\section{A MCGDM method with LNNs based on fuzzy measure and bidirectional projection measure}

In this section, we propose a novel method to solve MCGDM problems with LNNs based on the bidirectional projection method.

Let $A=\left\{A_{1}, A_{2}, \ldots, A_{m}\right\}$ be a set of alternatives, $C=\left\{C_{1}, C_{2}, \ldots ., C_{n}\right\}$ be the set of criteria, $D=\left\{D_{1}, D_{2}, \ldots, D_{p}\right\}$ be the set of DMs. Suppose that $y_{i j}^{k}=\left(g_{o_{i j}}^{k}, g_{q_{i j}}^{k}, g_{u_{i j}}^{k}\right)$ is a LNN which represents the evaluation information of the alternative $A_{i}$ with respect to the criterion $C_{j}$ given by the DM $D_{k} . Y^{k}=\left[y_{i j}^{k}\right]_{m \times n}$ is the decision matrix. $\lambda=\left(\lambda_{1}, \lambda_{2}, \ldots ., \lambda_{p}\right)^{T}$ is the weight vector of $\operatorname{DMs}_{k}(k=1,2, \ldots ., p)$, which satisfies $\lambda_{k} \in[0,1]$ and $\sum_{k=1}^{p} \lambda_{k}=1$. Here we assume the weight vector $w=\left(w_{1}, w_{2}, \cdots, w_{n}\right)$ of the criteria is partial known, and $w_{j} \in\left[a_{j}, b_{j}\right], a_{j}, b_{j} \in[0,1]$. Now, we can propose a novel MCGDM method by above information as follows:

Step 1: Normalize the decision-making information.

There usually are two types of evaluation criteria, i.e., benefit criteria and cost criteria. We should convert the different types of criteria to same type. That is, normalize each decision matrix $Y^{k}=\left[y_{i j}^{k}\right]_{m \times n}$ $(k=1,2, \ldots ., p)$ into the transformed decision matrix $R^{k}=\left[r_{i j}^{k}\right]_{m \times n}$ $(k=1,2, \ldots ., p)$, where $r_{i j}^{k}=\left(g_{\alpha_{i j}}^{k}, g_{\beta_{i j}}^{k}, g_{\gamma_{i j}}^{k}\right)$ and

$r_{i j}^{k}=\left(g_{\alpha_{i j}}^{k}, g_{\beta_{i j}}^{k}, g_{\gamma_{i j}}^{k}\right)=\left\{\begin{array}{l}\left(g_{o_{i j}}^{k}, g_{q_{i j}}^{k}, g_{u_{i j}}^{k}\right) \text { for the benefit criterion } C_{j} \\ \left(g_{u_{i j}}^{k}, g_{2 t-q_{i j}}^{k}, g_{o i j}^{k}\right) \text { for the } \cos t \text { criterion } C_{j}\end{array}\right.$ 
$k=1,2, \ldots ., p ; i=1,2, \ldots, m ; j=1,2, \ldots ., n$

Step 2: Obtain collective evaluation values.

The collective decision-making matrix $R=\left[r_{i j}\right]_{m \times n}=$ $\left(g_{\alpha_{i j}}, g_{\beta_{i j}}, g_{\gamma_{i j}}\right)_{m \times n}$ can be obtained by utilizing the LNWAA operator or LNWGA operator in Definition 8 or Definition 9.

Step 3: Calculate the Shapley weight of the criteria.

Firstly, use the maximum deviation model to get the fuzzy density $\mu\left(C_{j}\right)$ of criterion $C_{j}$. Then the $\lambda$-fuzzy measure of criteria can be obtained by formula (12), and the Shapley weight of each criterion can be calculated by formula (13).

Step 4: Determine the ideal alternative $R^{*}$.

For each criterion $C_{j}(j=1,2, \cdots, n)$, we can obtain the maximum value according to the comparison method in Definition 6, i.e., $r^{+}=\left(r_{1}^{+}, r_{2}^{+}, \cdots, r_{n}^{+}\right), r_{j}^{+}=\max \left(r_{i j}\right)(j=1,2, \cdots, n)$. Thus the ideal alternative is $R^{*}=\left(r_{1}^{+}, r_{2}^{+}, \cdots, r_{n}^{+}\right)$.

Step 5: Compute the bidirectional projection measure between $R^{*}$ and $R_{i}(i=1,2, \ldots, m)$.

$B P_{r o j}\left(R^{*}, R_{i}\right)=\frac{\left\|R^{*}\right\|\left\|R_{i}\right\|}{\left\|R^{*}\right\|\left\|R_{i}\right\|+\left|\left\|R^{*}\right\|-\left\|R_{i}\right\|\right| R^{*} \cdot R_{i}}$

where $\quad\left\|R^{*}\right\|=\sqrt{\sum_{j=1}^{n}\left(w_{j}\right)^{2}\left[\left(\frac{\alpha_{i j}^{*}}{2 t}\right)^{2}+\left(\frac{\beta_{i j}^{*}}{2 t}\right)^{2}+\left(\frac{\gamma_{i j}^{*}}{2 t}\right)^{2}\right]}, \quad\left\|R_{i}\right\|=$ $\sqrt{\sum_{j=1}^{n}\left(w_{j}\right)^{2}\left[\left(\frac{\alpha_{i j}}{2 t}\right)^{2}+\left(\frac{\beta_{i j}}{2 t}\right)^{2}+\left(\frac{\gamma_{i j}}{2 t}\right)^{2}\right]}$,

$R^{*} \cdot R_{i}=\sum_{j=1}^{n}\left(w_{j}\right)^{2}\left[\frac{\alpha_{i j}^{*}}{2 t} \cdot \frac{\alpha_{i j}}{2 t}+\frac{\beta_{i j}^{*}}{2 t} \cdot \frac{\beta_{i j}}{2 t}+\frac{\gamma_{i j}^{*}}{2 t} \cdot \frac{\gamma_{i j}}{2 t}\right]$.

Step 6: Rank the alternatives.

Rank the alternatives according to the values of $B P_{r o j}\left(R^{*}, R_{i}\right)$. The bigger the global value $B P_{r o j}\left(R^{*}, R_{i}\right)$, the better the alternative $A_{i}$.

\section{Application example}

In this section, we will illustrate the proposed MCGDM method in detail by some examples, and further prove its effectiveness and advantages by comparing with the existing MCGDM methods (Fang \& Ye, 2017; Liang et al., 2017; Tu, Ye, \& Wang, 2018; Ye, 2017b).

\subsection{The application of the proposed method}

In the following, we use a practical example from Liang et al. (2017) to demonstrate the MCGDM process of the proposed method.

Example 5.1. There are four companies $A_{1}, A_{2}, A_{3}, A_{4}$ as alternatives and there are five evaluation criteria: (1) $C_{1}$ is the geographic risk; (2) $C_{2}$ is the technological risk; (3) $C_{3}$ is the market risk; (4) $C_{4}$ is the management risk; (5) $C_{5}$ is the political risk, which are used to evaluate the alternatives by three DMs $D_{h}(h=1,2,3)$ based on the LTSs: $G=\left\{g_{i} \mid i \in[0,8]\right\}, \quad$ where $G=\left\{g_{0}=\right.$ extremely low, $g_{1}=$ pretty low, $g_{2}=$ low, $g_{3}=$ slightly low, $g_{4}=$ medium, $g_{5}=$ slightly high, $g_{6}=$ high ,

$g_{7}=$ pretty high, $g_{8}=$ perfect $\}$. Assume the weight vector of three DMs is $\lambda=\left(\frac{1}{3}, \frac{1}{3}, \frac{1}{3}\right)^{T}$, the weight vector of criteria is partly known and is expressed by $W_{C_{1}}=[0.05,0.15], W_{C_{2}}=[0.2,0.25], \quad W_{C_{3}}=$ $[0.15,0.25], W_{C_{4}}=[0.1,0.3], W_{C_{5}}=[0.2,0.3]$. The evaluation value of the alternative $A_{i}(i=1,2,3,4)$ on the criterion $C_{j}(j=1,2,3)$ by DM $D_{h}(h=1,2,3)$ is expressed by the LNN, and then three LNN decision matrices $Y^{h}=\left[y_{i j}^{h}\right]_{m \times n}$ are constructed and listed in Tables $1-3$. By the above information, it is necessary to give the ranking of the alternatives.

Case 1:. If the criteria weights are partial known, the decision-making steps of the proposed method as follows:

Step 1: Normalize the decision-making information.

Since all the criteria values belong to the cost type, we normalize evaluation values by Eq. (26), which are listed in Tables 4-6

Step 2: Obtain collective evaluation values.

Utilize the LNWAA operator in (10) to aggregate all individual decision matrix $R^{k} \quad(k=1,2, \ldots ., p)$ into collective one $R=\left[r_{i j}\right]_{m \times n}=\left(g_{\alpha_{i j}}, g_{\beta_{i j}}, g_{\gamma_{i j}}\right)_{m \times n}$, which is shown in Table 7 .

Step 3: Calculate the Shapley weight of the criteria.

Firstly, establish the maximum deviation model based on the fuzzy measure as follows:

$$
\begin{aligned}
\max D(\varphi)= & -0.3668\left(\mu\left(C_{1}\right)-\mu\left(C_{2}, C_{3}, C_{4}, C_{5}\right)\right) \\
& -0.1340\left(\mu\left(C_{2}\right)-\mu\left(C_{1}, C_{3}, C_{4}, C_{5}\right)\right)
\end{aligned}
$$

$-0.1419\left(\mu\left(C_{3}\right)-\mu\left(C_{1}, C_{2}, C_{4}, C_{5}\right)\right)+0.2692\left(\mu\left(C_{4}\right)-\mu\left(C_{1}, C_{2}, C_{3}, C_{5}\right)\right)$

$+0.3736\left(\mu\left(C_{5}\right)-\mu\left(C_{1}, C_{2}, C_{3}, C_{4}\right)\right)-0.1669\left(\mu\left(C_{1}, C_{2}\right)-\mu\left(C_{3}, C_{4}, C_{5}\right)\right)$

$-0.1696\left(\mu\left(C_{1}, C_{3}\right)-\mu\left(C_{2}, C_{4}, C_{5}\right)\right)-0.0325\left(\mu\left(C_{1}, C_{4}\right)-\mu\left(C_{2}, C_{3}, C_{5}\right)\right)$

$-0.0023\left(\mu\left(C_{1}, C_{5}\right)-\mu\left(C_{2}, C_{3}, C_{4}\right)\right)-0.0920\left(\mu\left(C_{2}, C_{3}\right)-\mu\left(C_{1}, C_{4}, C_{5}\right)\right)$

$+0.0451\left(\mu\left(C_{2}, C_{4}\right)-\mu\left(C_{1}, C_{3}, C_{5}\right)\right)+0.0798\left(\mu\left(C_{2}, C_{5}\right)-\mu\left(C_{1}, C_{3}, C_{4}\right)\right)$

$+0.2143\left(\mu\left(C_{3}, C_{4}\right)-\mu\left(C_{1}, C_{2}, C_{5}\right)\right)+0.0772\left(\mu\left(C_{3}, C_{4}\right)-\mu\left(C_{1}, C_{2}, C_{5}\right)\right)$ $+0.2143\left(\mu\left(C_{4}, C_{5}\right)-\mu\left(C_{1}, C_{2}, C_{3}\right)\right)+2.5962$

s.t. $\left\{\begin{array}{c}\mu\left(C_{1}\right) \in[0,0.1], \mu\left(C_{2}\right) \in[0.2,0.25], \mu\left(C_{3}\right) \in[0.15,0.25], \mu\left(C_{4}\right) \\ \in[0.1,0.3], \mu\left(C_{5}\right) \in[0.2,0.3] \\ \mu(\phi)=0, \mu\left(C_{1}, C_{2}, C_{3}, C_{4}, C_{5}\right)=1 \\ \mu(S) \leqslant \mu(T), \forall S, T \subseteq C, S \subseteq T\end{array}\right.$

Then apply the Lingo tool to solve this linear programming model. Thus we get the fuzzy density $\mu\left(C_{j}\right)$ of criteria $C_{j}$ as follows: $\mu\left(C_{1}\right)=0.05, \mu\left(C_{2}\right)=0.25, \mu\left(C_{3}\right)=0.15, \mu\left(C_{4}\right)=\mu\left(C_{5}\right)=0.3$.

Then we can get $\lambda=-0.1225$ by formula (12), and the $\lambda$-fuzzy measure of the criteria can be calculated and listed in Table 8 .

Based on the calculated $\lambda$-fuzzy measure, we obtain the Shapley weight of each criterion according to formula (13).

$\varphi_{1}=0.05 \varphi_{2}=0.24 \varphi_{3}=0.15 \varphi_{4}=0.28 \varphi_{5}=0.28$

Step 4: Determine the ideal alternative $R^{*}$.

According to the comparison method in Definition 6, we can obtain the maximum value of each criterion in Table 9.

Thus we identify the ideal alternative $R^{*}=\left(r_{1}^{+}, r_{2}^{+}, \cdots, r_{5}^{+}\right)$.

Step 5: Calculate the bidirectional projection measure between $R^{*}$ and $R_{i}(i=1,2,3,4)$ based on the formula (27).

The modules of $R^{*}$ and $R_{i}(i=1,2,3,4)$ are $\left\|R^{*}\right\|=0.273$, $\left\|R_{1}\right\|=0.343,\left\|R_{2}\right\|=0.371,\left\|R_{3}\right\|=0.336,\left\|R_{4}\right\|=0.280$ respectively.

The inner products of $R^{*}$ and $R_{i}(i=1,2,3,4)$ are $R^{*} \cdot R_{1}=0.098$, $R^{*} \cdot R_{2}=0.174, R^{*} \cdot R_{3}=0.197, R^{*} \cdot R_{4}=0.167$.

The bidirectional projection measures between $R^{*}$ and $R_{i}$ $(i=1,2,3,4)$ are

$$
B P_{r o j}\left(R^{*}, R_{1}\right)=0.931 B P_{r o j}\left(R^{*}, R_{2}\right)=0.855 B P_{r o j}\left(R^{*}, R_{3}\right)=0.880 B P_{r o j}\left(R^{*}, R_{4}\right)
$$$$
=0.984
$$ 
Table 1

LNN decision matrix $Y^{1}$ given by DM $D_{1}$.

\begin{tabular}{|c|c|c|c|c|c|}
\hline & $C_{1}$ & $C_{2}$ & $C_{3}$ & $C_{4}$ & $C_{5}$ \\
\hline$A_{1}$ & $\left(g_{1.0}, g_{2.0}, g_{1.0}\right)$ & $\left(g_{2.0}, g_{3.0}, g_{2.0}\right)$ & $\left(g_{4.0}, g_{4.0}, g_{3.0}\right)$ & $\left(g_{1.0}, g_{5.0}, g_{1.0}\right)$ & $\left(g_{3.0}, g_{3.0}, g_{2.0}\right)$ \\
\hline$A_{2}$ & $\left(g_{2.0}, g_{6.0}, g_{2.0}\right)$ & $\left(g_{3.0}, g_{8.0}, g_{2.0}\right)$ & $\left(g_{2.0}, g_{4.0}, g_{1.0}\right)$ & $\left(g_{3.0}, g_{1.0}, g_{2.0}\right)$ & $\left(g_{1.0}, g_{2.0}, g_{1.0}\right)$ \\
\hline$A_{3}$ & $\left(g_{2.0}, g_{3.0}, g_{1.0}\right)$ & $\left(g_{3.0}, g_{2.0}, g_{3.0}\right)$ & $\left(g_{1.0}, g_{4.0}, g_{1.0}\right)$ & $\left(g_{3.0}, g_{5.0}, g_{1.0}\right)$ & $\left(g_{5.0}, g_{2.0}, g_{4.0}\right)$ \\
\hline$A_{4}$ & $\left(g_{3.0}, g_{1.0}, g_{2.0}\right)$ & $\left(g_{1.0}, g_{7.0}, g_{1.0}\right)$ & $\left(g_{4.0}, g_{6.0}, g_{3.0}\right)$ & $\left(g_{2.0}, g_{5.0}, g_{1.0}\right)$ & $\left(g_{4.0}, g_{6.0}, g_{4.0}\right)$ \\
\hline
\end{tabular}

Step 6: Rank the alternatives.

Since $B P_{r o j}\left(R^{*}, R_{4}\right)>B P_{r o j}\left(R^{*}, R_{1}\right)>B P_{r o j}\left(R^{*}, R_{3}\right)>B P_{r o j}\left(R^{*}, R_{2}\right)$, we can obtain $A_{4}>A_{1}>A_{3}$

$>A_{2}$ and the best choice is $A_{4}$.

Case 2:. If the criteria weights are given directly by DMs, for example, the weight vector is $\omega_{j}=(0.19,0.21,0.15,0.05,0.4)^{T}$, then we can skip the Step 3 of the proposed method to handle the above problem.

Then, the bidirectional projection measures between $R^{*}$ and $R_{i}$ $(i=1,2,3,4)$ of Case 2 can be calculated as follows: $B P_{\text {roj }}\left(R^{*}, R_{1}\right)=0.933, \quad B P_{\text {roj }}\left(R^{*}, R_{2}\right)=0.965, \quad B P_{\text {roj }}\left(R^{*}, R_{3}\right)=0.938$, $B P_{r o j}\left(R^{*}, R_{4}\right)=0.999$. So we have $A_{4}>A_{3}>A_{1}>A_{2}$, which is different from the ranking result of Case 1 because of the diverse weights of criteria values.

In decision making process, weights of criteria will influence the final decision-making result. However, it is difficult for DMs to assign appropriate criteria weights due to the time pressure, data loss, and limited domain knowledge about the problem. Furthermore, it is common that there are interactions among criteria in realistic decision environment. To get the reasonable criteria weights, we combine the maximum deviation model and fuzzy measure, and propose Shapley weight which not only avoids the subjective judgments of DMs but also considers the relationship between criteria. The criteria weights in Case 2 are given only from the preference or judgments of DMs while the Case 1 uses the maximum deviation model to determine the criteria weights, which can effectively reduce the subjective effects. Therefore, the weight model in the paper is more reasonable and suitable to determine weight for each criterion.

\subsection{The verification of the effectiveness}

In the following, we give an example to compare the ranking results of the proposed MCGDM method with the ones of Fang and Ye's method (Fang \& Ye, 2017) based on the LNWAA operator and Liang et al.'s method (Liang et al., 2017) based on the extended TOPSIS model.

Example 5.2. An investment company needs to select an industry to invest and there are four possible investment alternatives $\left\{A_{1}, A_{2}, A_{3}, A_{4}\right\}$ which are the technology company, the jewelry company, the food company, the sporting goods company, respectively. There are three criteria (suppose their weight vector is $\left.W=(0.35,0.25,0.4)^{T}\right)$ : the market risk $\left(C_{1}\right)$, the growth risk $\left(C_{2}\right)$, the policy risk $\left(C_{3}\right)$. The DM gives the evaluation information of the alternatives $\left\{A_{1}, A_{2}, A_{3}, A_{4}\right\}$ with the criteria $C_{j}(j=1,2,3)$ by LNNs based on the LTs: $\quad G=\left\{g_{0}=\right.$ extremely low, $g_{1}=$ pretty low, $g_{2}=$ low, $g_{3}=$ slight ly low, $g_{4}=$ medium, $g_{5}=$ slightly high, $g_{6}=$ high,$g_{7}=$ pretty .

high, $g_{8}=$ perfect $\}$

Table 2

LNN decision matrix $Y^{2}$ given by DM $D_{2}$.

\begin{tabular}{|c|c|c|c|c|c|}
\hline & $C_{1}$ & $C_{2}$ & $C_{3}$ & $C_{4}$ & $C_{5}$ \\
\hline$A_{1}$ & $\left(g_{1.0}, g_{6.0}, g_{1.0}\right)$ & $\left(g_{4.0}, g_{3.0}, g_{4.0}\right)$ & $\left(g_{2.0}, g_{6.0}, g_{2.0}\right)$ & $\left(g_{3.0}, g_{5.0}, g_{2.0}\right)$ & $\left(g_{5.0}, g_{2.0}, g_{4.0}\right)$ \\
\hline$A_{2}$ & $\left(g_{1.0}, g_{4.0}, g_{1.0}\right)$ & $\left(g_{3.0}, g_{2.0}, g_{1.0}\right)$ & $\left(g_{2.0}, g_{3.0}, g_{4.0}\right)$ & $\left(g_{4.0}, g_{0.0}, g_{5.0}\right)$ & $\left(g_{2.0}, g_{6.0}, g_{4.0}\right)$ \\
\hline$A_{3}$ & $\left(g_{3.0}, g_{5.0}, g_{2.0}\right)$ & $\left(g_{2.0}, g_{4.0}, g_{3.0}\right)$ & $\left(g_{1.0}, g_{6.0}, g_{5.0}\right)$ & $\left(g_{3.0}, g_{5.0}, g_{3.0}\right)$ & $\left(g_{2.0}, g_{6.0}, g_{1.0}\right)$ \\
\hline$A_{4}$ & $\left(g_{2.0}, g_{7.0}, g_{2.0}\right)$ & $\left(g_{4.0}, g_{6.0}, g_{1.0}\right)$ & $\left(g_{3.0}, g_{7.0}, g_{2.0}\right)$ & $\left(g_{4.0}, g_{4.0}, g_{2.0}\right)$ & $\left(g_{3.0}, g_{8.0}, g_{4.0}\right)$ \\
\hline
\end{tabular}

The decision matrix $X=\left[x_{i j}\right]_{4 \times 3}$ is listed in Table 10 .

For convenience, we set the same weight vector of the criteria $W=(0.35,0.25,0.4)^{T}$ for these methods and the comparison results are shown in Table 11.

From Table 11, we can see that the ranking result of proposed method is same as the ones of the methods in Fang and Ye (2017) and Liang et al. (2017), i.e., $A_{4}>A_{1}>A_{2}>A_{3}$. It can prove our proposed method is effective and rational.

\subsection{Further comparison with other methods}

In the above sub-section, we have proved the validity of our proposed method by obtaining the same ranking results with the two existing methods (Fang \& Ye, 2017; Liang et al., 2017). In the following, we will set up two examples to further illustrate the advantages of the proposed method. Example 5.3 will show the advantages of the proposed method based on the bidirectional projection by comparing with projection of LNNs and the method based on TOPSIS model proposed by Liang et al. (2017). Example 5.4 will show the advantages of the proposed method with LNNs by comparing with bidirectional projection of SVNs in Ye (2017b) and the weighted symmetry measure of SVNs in Tu et al. (2018).

Example 5.3. A company plans to develop a new career and there are four possible alternatives and there are five criteria shown as follows (suppose weight vector is $\left.W=(0.05,0.24,0.15,0.28,0.28)^{T}\right)$ : the production $\left(C_{1}\right)$, the technical skill $\left(C_{2}\right)$, the management development $\left(C_{3}\right)$, the market growth $\left(C_{4}\right)$, the social policy $\left(C_{5}\right)$. The DM gives the evaluation information of the alternatives $\left\{A_{1}, A_{2}, A_{3}, A_{4}\right\}$ with the criteria $C_{j}(j=1,2,3,4,5)$ by LNNs based on the LTs: $G=\left\{g_{0}=\right.$ extremely bad, $g_{1}=$ pretty bad, $g_{2}=$ bad, $g_{3}=$ a little

bad, $g_{4}=$ medium, $g_{5}=$ a little good, $g_{6}=$ good, $g_{7}=$ pretty good, $g_{8}=$ perfect $\}$. The decision matrix $X=\left[x_{i j}\right]_{4 \times 5}$ is listed in Table 12. Then we can get the ranking results in Table 13.

From Table 13, it can be seen that the ranking results of the proposed method based on the bidirectional projection is different from the ones by projection and TOPSIS (Liang et al., 2017). Next, we discuss and analyze the superiority of our proposed method as follows:

(1) Compared with the method based on the projection measure

The projection measure can be calculated by formula $P_{\text {roj }}\left(A^{*}, A_{i}\right)=\frac{A^{*} \cdot A_{i}}{\left\|A^{*}\right\|}$

where $A^{*}=\left(x_{1}^{+}, x_{2}^{+}, x_{3}^{+}, x_{4}^{+}\right), x_{j}^{+}=\max \left(x_{i j}^{*}\right)(j=1,2,3,4)$. Then the ideal alternative is $A^{*}=A_{4}$ and we can $\operatorname{get} P_{r o j} A^{*}\left(A_{1}\right)=0.297$, 
Table 3

LNN decision matrix $Y^{3}$ given by DM $D_{3}$.

\begin{tabular}{|c|c|c|c|c|c|}
\hline & $C_{1}$ & $C_{2}$ & $C_{3}$ & $C_{4}$ & $C_{5}$ \\
\hline$A_{1}$ & $\left(g_{2.0}, g_{4.0}, g_{1.0}\right)$ & $\left(g_{3.0}, g_{5.0}, g_{2.0}\right)$ & $\left(g_{5.0}, g_{1.0}, g_{4.0}\right)$ & $\left(g_{2.0}, g_{6.0}, g_{1.0}\right)$ & $\left(g_{3.0}, g_{3.0}, g_{2.0}\right)$ \\
\hline$A_{2}$ & $\left(g_{1.0}, g_{2.0}, g_{1.0}\right)$ & $\left(g_{2.0}, g_{4.0}, g_{2.0}\right)$ & $\left(g_{1.0}, g_{5.0}, g_{3.0}\right)$ & $\left(g_{4.0}, g_{2.0}, g_{0.0}\right)$ & $\left(g_{0.0}, g_{5.0}, g_{6.0}\right)$ \\
\hline$A_{3}$ & $\left(g_{2.0}, g_{3.0}, g_{3.0}\right)$ & $\left(g_{1.0}, g_{5.0}, g_{2.0}\right)$ & $\left(g_{2.0}, g_{4.0}, g_{5.0}\right)$ & $\left(g_{0.0}, g_{4.0}, g_{6.0}\right)$ & $\left(g_{3.0}, g_{2.0}, g_{4.0}\right)$ \\
\hline$A_{4}$ & $\left(g_{2.0}, g_{3.0}, g_{2.0}\right)$ & $\left(g_{4.0}, g_{2.0}, g_{1.0}\right)$ & $\left(g_{1.0}, g_{4.0}, g_{3.0}\right)$ & $\left(g_{3.0}, g_{4.0}, g_{5.0}\right)$ & $\left(g_{0.0}, g_{4.0}, g_{5.0}\right)$ \\
\hline
\end{tabular}

Table 4

Normalized decision matrix $R^{1}$ by DM $D_{1}$.

\begin{tabular}{llllll}
\hline & $C_{1}$ & $C_{2}$ & $C_{3}$ & $C_{4}$ & $C_{5}$ \\
\hline$A_{1}$ & $\left(g_{1}, g_{6}, g_{1}\right)$ & $\left(g_{2}, g_{5}, g_{2}\right)$ & $\left(g_{3}, g_{4}, g_{4}\right)$ & $\left(g_{1}, g_{3}, g_{1}\right)$ & $\left(g_{2}, g_{5}, g_{3}\right)$ \\
$A_{2}$ & $\left(g_{2}, g_{2}, g_{2}\right)$ & $\left(g_{2}, g_{0}, g_{3}\right)$ & $\left(g_{1}, g_{4}, g_{2}\right)$ & $\left(g_{2}, g_{7}, g_{3}\right)$ & $\left(g_{1}, g_{6}, g_{1}\right)$ \\
$A_{3}$ & $\left(g_{1}, g_{5}, g_{2}\right)$ & $\left(g_{3}, g_{6}, g_{3}\right)$ & $\left(g_{1}, g_{4}, g_{1}\right)$ & $\left(g_{1}, g_{3}, g_{3}\right)$ & $\left(g_{4}, g_{6}, g_{5}\right)$ \\
$A_{4}$ & $\left(g_{2}, g_{7}, g_{3}\right)$ & $\left(g_{1}, g_{1}, g_{1}\right)$ & $\left(g_{3}, g_{2}, g_{4}\right)$ & $\left(g_{1}, g_{3}, g_{2}\right)$ & $\left(g_{4}, g_{2}, g_{4}\right)$ \\
\hline
\end{tabular}

Table 5

Normalized decision matrix $R^{2}$ by DM $D_{2}$.

\begin{tabular}{llllll}
\hline & $C_{1}$ & $C_{2}$ & $C_{3}$ & $C_{4}$ & $C_{5}$ \\
\hline$A_{1}$ & $\left(g_{1}, g_{2}, g_{1}\right)$ & $\left(g_{4}, g_{5}, g_{4}\right)$ & $\left(g_{2}, g_{2}, g_{2}\right)$ & $\left(g_{2}, g_{3}, g_{3}\right)$ & $\left(g_{4}, g_{6}, g_{5}\right)$ \\
$A_{2}$ & $\left(g_{1}, g_{4}, g_{1}\right)$ & $\left(g_{1}, g_{6}, g_{3}\right)$ & $\left(g_{4}, g_{5}, g_{2}\right)$ & $\left(g_{5}, g_{8}, g_{4}\right)$ & $\left(g_{4}, g_{2}, g_{2}\right)$ \\
$A_{3}$ & $\left(g_{2}, g_{3}, g_{3}\right)$ & $\left(g_{3}, g_{4}, g_{2}\right)$ & $\left(g_{5}, g_{2}, g_{1}\right)$ & $\left(g_{3}, g_{3}, g_{3}\right)$ & $\left(g_{1}, g_{2}, g_{2}\right)$ \\
$A_{4}$ & $\left(g_{2}, g_{1}, g_{2}\right)$ & $\left(g_{1}, g_{2}, g_{4}\right)$ & $\left(g_{2}, g_{1}, g_{3}\right)$ & $\left(g_{2}, g_{4}, g_{4}\right)$ & $\left(g_{4}, g_{0}, g_{3}\right)$ \\
\hline
\end{tabular}

Table 6

Normalized decision matrix $R^{3}$ by DM $D_{3}$.

\begin{tabular}{llllll}
\hline & $C_{1}$ & $C_{2}$ & $C_{3}$ & $C_{4}$ & $C_{5}$ \\
\hline$A_{1}$ & $\left(g_{1}, g_{4}, g_{2}\right)$ & $\left(g_{2}, g_{3}, g_{3}\right)$ & $\left(g_{4}, g_{7}, g_{5}\right)$ & $\left(g_{1}, g_{2}, g_{2}\right)$ & $\left(g_{2}, g_{5}, g_{3}\right)$ \\
$A_{2}$ & $\left(g_{1}, g_{6}, g_{1}\right)$ & $\left(g_{2}, g_{4}, g_{2}\right)$ & $\left(g_{3}, g_{3}, g_{1}\right)$ & $\left(g_{0}, g_{6}, g_{4}\right)$ & $\left(g_{6}, g_{3}, g_{0}\right)$ \\
$A_{3}$ & $\left(g_{3}, g_{5}, g_{2}\right)$ & $\left(g_{2}, g_{3}, g_{1}\right)$ & $\left(g_{5}, g_{4}, g_{2}\right)$ & $\left(g_{6}, g_{4}, g_{0}\right)$ & $\left(g_{4}, g_{6}, g_{3}\right)$ \\
$A_{4}$ & $\left(g_{2}, g_{5}, g_{2}\right)$ & $\left(g_{1}, g_{6}, g_{4}\right)$ & $\left(g_{3}, g_{4}, g_{1}\right)$ & $\left(g_{5}, g_{4}, g_{3}\right)$ & $\left(g_{5}, g_{4}, g_{0}\right)$ \\
\hline
\end{tabular}

$P_{\text {roj } A^{*}}\left(A_{2}\right)=0.193, \quad P_{\text {roj } A^{*}}\left(A_{3}\right)=0.278, \quad P_{\text {roj } A^{*}}\left(A_{4}\right)=0.265 . \quad$ Because $P_{\text {roja }}\left(A_{1}\right)>P_{\text {roja }}\left(A_{3}\right)>P_{\text {rojA }} *\left(A_{4}\right)>P_{\text {roja }} *\left(A_{2}\right)$, we have $A_{1}>A_{3}>A_{4}>A_{2}$.

Clearly, $P_{r o j} A^{*}\left(A_{1}\right)$ and $P_{r o j} A^{*}\left(A_{3}\right)$ is larger than $P_{r o j} A^{*}\left(A_{4}\right)$. In fact, $A_{4}$ is closer to $A^{*}$ than $A_{1}$ and $A_{3}$ due to $A^{*}=A_{4}$. Thus the ranking result obtained by using the projection measure is unreasonable. On the other hand, the bidirectional projection measure is based on the projection of the $A_{i}$ on the $A^{*} P_{r o j} A^{*}\left(A_{i}\right)$ and the projection of the $A^{*}$ on the $A_{i}$ $P_{r o j A_{i}}\left(A^{*}\right)$. Due to the difference between the $P_{r o j} A^{*}\left(A_{i}\right)$ and the $P_{\text {roj } A_{i}}\left(A^{*}\right)$, we utilize the closeness degree $\left|P_{\text {roj } A^{*}}\left(A_{i}\right)-P_{\text {roj } A_{i}}\left(A^{*}\right)\right|$ to select the optimal alternative. The closer to $A^{*}$, the better $A_{i}$ is. Since $P_{\text {roj } A^{*}}\left(A_{4}\right)-P_{\text {roj } A_{4}}\left(A^{*}\right) \mid=0<$

$\left|P_{\text {roj } A^{*}}\left(A_{2}\right)-P_{\text {roj } A_{2}}\left(A^{*}\right)\right|=0.0298<\left|P_{\text {roj }} A^{*}\left(A_{3}\right)-P_{\text {roj } A_{1}}\left(A^{*}\right)\right|=0.0327<\mid P_{\text {roj }} A^{*}\left(A_{1}\right)$, $-P_{\text {roj } A_{2}}\left(A^{*}\right) \mid=0.1197$

then $B P_{r o j}\left(R^{*}, R_{4}\right)=1>B P_{r o j}\left(R^{*}, R_{2}\right)=0.9710>B P_{r o j}\left(R^{*}, R_{3}\right)=0.9683>$ $B P_{\text {roj }}\left(R^{*}, R_{1}\right)=0.8931$, which is more reasonable and comprehensive than the general projection measure based on the single directional projection magnitude between $A_{i}$ and $A^{*}$.

Comparing with the projection model, the proposed bidirectional projection method not only considers the distance and included angle but also the bidirectional projection magnitudes. Therefore, the bidirectional projection measure is more general and reasonable than the projection method because it can overcome the shortcoming of the projection method. In addition, the value of bidirectional projection measure is normalized within $[0,1]$ to avoid some unreasonable result.

(2) Compared with the method based on the LNN-TOPSIS (Liang et al., 2017)

As we have known, the TOPSIS model determines the best alternative which has the shortest distance from the ideal alternative and the farthest distance from the negative ideal alternative. The proposed method selects the optimal alternative considering both the distance and the included angle between options, which can better reflect superiority and rationality of ranking result. For example, comparing with the bidirectional projection measure, the differences of the ranking result between TOPSIS method in Liang et al. (2017) and the proposed method are $A_{2}$ and $A_{3}$. Then we calculate the distance between ideal alternative $A^{+}$and $A_{i}$ is $d_{4}^{+}=0<d_{3}^{+}=0.70<d_{2}^{+}=0.71<d_{1}^{+}=1.36$, and the distance between negative ideal alternative $A^{-}$and $A_{i}$ is $d_{4}^{-}=1.36>d_{2}^{-}=0.72>d_{3}^{-}=0.70>d_{1}^{-}=0$. Then the associated correlation coefficients are $D_{4}=1>D_{2} \approx D_{3}=0.50>D_{1}=0$. We can see that it is hard to distinguish which is preferable if the distance between $A^{+}$and $A_{i}$ is equal to the distance between $A^{-}$and $A_{i}$. In other word, there are defects in TOPSIS model when $d_{i}^{+}=d_{i}^{-}$. In such case, the bidirectional projection measure is more reasonable because it considers both distance and included angle or even the bidirectional projection magnitudes. So our proposed method is more effective and suitable than Liang et al.'s method (Liang et al., 2017) in practical applications.

Example 5.4. An investment company plans to invest a domestic coal mine and there are four possible mines as alternatives. There are five criteria shown as follows (suppose their weight vector is $\left.W=(0.05,0.24,0.15,0.28,0.28)^{T}\right)$ : the geological condition $\left(C_{1}\right)$, the production $\left(C_{2}\right)$, the market development $\left(C_{3}\right)$, the technical capacity $\left(C_{4}\right)$, the social policy $\left(C_{5}\right)$. The DM gives the evaluation information of the alternatives $\left\{A_{1}, A_{2}, A_{3}, A_{4}\right\}$ with the criteria $C_{j}(j=1,2,3,4,5)$ by LNNs based on the LTs: $G=\left\{g_{0}=\right.$ extremely bad, $g_{1}=$ pretty bad, $g_{2}=$ bad, $g_{3}=$ a little bad, $g_{4}=$ medium, $g_{5}=$ a little good, $g_{6}=$ good, $g_{7}=$ pretty good, $g_{8}=$ perfect $\}$. The decision matrix $X=\left[x_{i j}\right]_{4 \times 5}$ is listed in Table 14, and the comparison results of different approaches are shown in Table 15.

From Table 15, it can be seen that the ranking result of the proposed method based on bidirectional projection with LNNs is different from

Table 7

Collective decision matrix $R$.

\begin{tabular}{|c|c|c|c|c|c|}
\hline & $C_{1}$ & $C_{2}$ & $C_{3}$ & $C_{4}$ & $C_{5}$ \\
\hline$A_{1}$ & $\left(g_{1.00}, g_{3.63}, g_{1.26}\right)$ & $\left(g_{2.76}, g_{4.22}, g_{2.89}\right)$ & $\left(g_{3.08}, g_{3.83}, g_{3.42}\right)$ & $\left(g_{1.35}, g_{2.62}, g_{1.82}\right)$ & $\left(g_{2.76}, g_{5.31}, g_{3.56}\right)$ \\
\hline$A_{2}$ & $\left(g_{1.35}, g_{3.63}, g_{1.26}\right)$ & $\left(g_{1.68}, g_{0.00}, g_{2.62}\right)$ & $\left(g_{2.81}, g_{3.91}, g_{1.59}\right)$ & $\left(g_{2.76}, g_{6.95}, g_{3.63}\right)$ & $\left(g_{4.17}, g_{3.30}, g_{0.00}\right)$ \\
\hline$A_{3}$ & $\left(g_{2.06}, g_{4.22}, g_{2.29}\right)$ & $\left(g_{2.69}, g_{4.16}, g_{1.82}\right)$ & $\left(g_{4.02}, g_{3.17}, g_{1.26}\right)$ & $\left(g_{3.88}, g_{3.30}, g_{0.00}\right)$ & $\left(g_{3.18}, g_{4.16}, g_{3.11}\right)$ \\
\hline$A_{4}$ & $\left(g_{2.00}, g_{3.27}, g_{2.29}\right)$ & $\left(g_{1.00}, g_{2.29}, g_{2.52}\right)$ & $\left(g_{2.69}, g_{2.00}, g_{2.29}\right)$ & $\left(g_{2.99}, g_{3.63}, g_{2.88}\right)$ & $\left(g_{4.37}, g_{0.00}, g_{0.00}\right)$ \\
\hline
\end{tabular}


Table 8

$\lambda$-fuzzy measure of criteria.

\begin{tabular}{|c|c|c|c|c|c|c|c|}
\hline Criteria & & Criteria & & Criteria & & Criteria & \\
\hline$\{\phi\}$ & 0 & $\left\{C_{1}, C_{4}\right\}$ & 0.348 & $\left\{C_{1}, C_{2}, C_{3}\right\}$ & 0.443 & $\left\{C_{2}, C_{4}, C_{5}\right\}$ & 0.821 \\
\hline$\left\{C_{1}\right\}$ & 0.05 & $\left\{C_{1}, C_{5}\right\}$ & 0.348 & $\left\{C_{1}, C_{2}, C_{4}\right\}$ & 0.588 & $\left\{C_{3}, C_{4}, C_{5}\right\}$ & 0.728 \\
\hline$\left\{C_{2}\right\}$ & 0.25 & $\left\{C_{2}, C_{3}\right\}$ & 0.395 & $\left\{C_{1}, C_{2}, C_{5}\right\}$ & 0.588 & $\left\{C_{1}, C_{2}, C_{3}, C_{4}\right\}$ & 0.727 \\
\hline$\left\{C_{3}\right\}$ & 0.15 & $\left\{C_{2}, C_{4}\right\}$ & 0.541 & $\left\{C_{1}, C_{3}, C_{4}\right\}$ & 0.492 & $\left\{C_{1}, C_{2}, C_{3}, C_{5}\right\}$ & 0.727 \\
\hline$\left\{C_{4}\right\}$ & 0.3 & $\left\{C_{2}, C_{5}\right\}$ & 0.541 & $\left\{C_{1}, C_{3}, C_{5}\right\}$ & 0.492 & $\left\{C_{1}, C_{2}, C_{4}, C_{5}\right\}$ & 0.866 \\
\hline$\left\{C_{5}\right\}$ & 0.3 & $\left\{C_{3}, C_{4}\right\}$ & 0.444 & $\left\{C_{1}, C_{4}, C_{5}\right\}$ & 0.635 & $\left\{C_{1}, C_{3}, C_{4}, C_{5}\right\}$ & 0.774 \\
\hline$\left\{C_{1}, C_{2}\right\}$ & 0.298 & $\left\{C_{3}, C_{5}\right\}$ & 0.444 & $\left\{C_{2}, C_{3}, C_{4}\right\}$ & 0.681 & $\left\{C_{2}, C_{3}, C_{4}, C_{5}\right\}$ & 0.956 \\
\hline$\left\{C_{1}, C_{3}\right\}$ & 0.199 & $\left\{C_{4}, C_{5}\right\}$ & 0.589 & $\left\{C_{2}, C_{3}, C_{5}\right\}$ & 0.681 & $\left\{C_{1}, C_{2}, C_{3}, C_{4}, C_{5}\right\}$ & 1 \\
\hline
\end{tabular}

Table 9

The maximum value of each criterion.

\begin{tabular}{|c|c|c|c|c|}
\hline$r_{1}^{+}$ & $r_{2}^{+}$ & $r_{3}^{+}$ & $r_{4}^{+}$ & $r_{5}^{+}$ \\
\hline$\left(g_{1.35}, g_{3.63}, g_{1.26}\right)$ & $\left(g_{1.68}, g_{0}, g_{2.62}\right)$ & $\left(g_{4.02}, g_{3.17}, g_{1.26}\right)$ & $\left(g_{3.88}, g_{3.3}, g_{0}\right)$ & $\left(g_{4.37}, g_{0}, g_{0}\right)$ \\
\hline
\end{tabular}

Table 10

Linguistic neutrosophic decision matrix of Example 5.2.

\begin{tabular}{llll}
\hline & $C_{1}$ & $C_{2}$ & $C_{3}$ \\
\hline$A_{1}$ & $\left(g_{6.0}, g_{1.0}, g_{2.0}\right)$ & $\left(g_{7.0}, g_{2.0}, g_{1.0}\right)$ & $\left(g_{5.0}, g_{2.0}, g_{3.0}\right)$ \\
$A_{2}$ & $\left(g_{7.0}, g_{2.0}, g_{2.0}\right)$ & $\left(g_{6.0}, g_{2.0}, g_{2.0}\right)$ & $\left(g_{6.0}, g_{2.0}, g_{2.0}\right)$ \\
$A_{3}$ & $\left(g_{6.0}, g_{2.0}, g_{2.0}\right)$ & $\left(g_{6.0}, g_{2.0}, g_{2.0}\right)$ & $\left(g_{6.0}, g_{3.0}, g_{2.0}\right)$ \\
$A_{4}$ & $\left(g_{7.0}, g_{1.0}, g_{2.0}\right)$ & $\left(g_{6.0}, g_{2.0}, g_{2.0}\right)$ & $\left(g_{6.0}, g_{2.0}, g_{1.0}\right)$ \\
\hline
\end{tabular}

Table 11

The ranking results by different approaches of Example 5.2.

\begin{tabular}{ll}
\hline Approaches & Ranking orders \\
\hline Approach with LNWAA operator (Fang \& Ye, 2017) & $A_{4}>A_{1}>A_{2}>A_{3}$ \\
Approach with LNN-TOPSIS (Liang et al., 2017) & $A_{4}>A_{1}>A_{2}>A_{3}$ \\
the proposed method based on the bidirectional projection & $A_{4}>A_{1}>A_{2}>A_{3}$ \\
\hline
\end{tabular}

the ones by Ye's method based on SVN-bidirectional projection (Ye, 2017b) and Tu et al.'s method based on SVN-symmetry measure (Tu et al., 2018). In the following, we give analysis about this experimental result as follows:

(1) Compared with the method (Ye, 2017b) based on the SVN-bidirectional projection

The information in Reference (Ye, 2017b) is SVNNs. To begin; we translate LNNs into SVNNs. There are two conversion methods by LSF in Peng and Wang (2016). For example, when $f=f_{1}\left(s_{i}\right)$, the evaluation value of $x_{12}=\left(g_{3.0}, g_{4.0}, g_{3.0}\right)$ is converted into $(0.375,0.5,0.375)$; when $f=f_{2}\left(s_{i}\right)(a=1.37)$, the evaluation value of $x_{12}$ is converted into $(0.427,0.5,0.427)$

Then we use the SVN-bidirectional projection method (Ye, 2017b) to rank the alternatives. From Table 13, we can see the differences of ranking results by the proposed method and the method in Ye (2017b) based on the LSF $f=f_{1}\left(s_{i}\right)$ are $A_{1}$ and $A_{3}$. The module of each
Table 13

The ranking results of different approaches of Example 5.3.

\begin{tabular}{ll}
\hline Approaches & Ranking orders \\
\hline Approach based on the projection with LNNs & $A_{1}>A_{3}>A_{4}>A_{2}$ \\
Approach with LNN-TOPSIS (Liang et al., 2017) & $A_{4}>A_{2} \approx A_{3}>A_{1}$ \\
The proposed method based on the bidirectional projection & $A_{4}>A_{2}>A_{3}>A_{1}$ \\
\hline
\end{tabular}

alternative based two methods is same, but the inner product between $A^{*}$ and $A_{i}$ is changed. The main reason is these two method adopt different ways to determine the ideal alternative: our method is based on the score function, while the method in Ye (2018) is determined by $A^{*}=\left(e_{1}^{*}, e_{2}^{*}, \cdots, e_{n}^{*}\right)$ and $e_{j}^{*}=\left(\max \left(T_{i j}\right), \min \left(I_{i j}\right), \min \left(F_{i j}\right)\right)$.

It is worth noting that the method in Ye $\stackrel{i}{i}(2017 \mathrm{~b})$ based on the $f=f_{2}\left(s_{i}\right)$ has the same ranking order as the proposed method, which reflects there exists inaccuracy in the conversion process of $f=f_{1}\left(s_{i}\right)$. The characteristics of two kinds of LSFs can be graphically shown in Fig. 1 (suppose $a=1.37$ ).

From Fig. 1, we can see LSF1 only shows the conversion values unidirectional growth. But in practical application, there are two directions including "good" and "bad" when DMs judge the criteria values. Thus, LSF2 is more suitable to reflect the psychological process of DMs by bidirectional geometric growth. Although LSF2 is a good tool in linguistic information transformation, LNNs can describe more complex linguistic information and is more suitable for qualitative decisionmaking environment than SVNs. So our proposed method with LNNs is more flexible and innovative.

(2) Compared with the method (Tu et al., 2018) based on the weighted symmetry measure between SVNSs

Similar to Ye's MCDM method (Ye, 2017b), the method in Tu et al. (2018) is also based on SVNNs so that we translate LNNs into SVNNs with $f_{2}\left(s_{i}\right)$. Then we calculate the weighted symmetry measure values between the ideal alternative $A^{*}$ and each alternative $A_{i}(i=1,2,3,4)$ and obtain the corresponding ranking order, i.e., $A_{4}>A_{1}>A_{3}>A_{2}$.

Table 12

Linguistic neutrosophic decision matrix of Example 5.3.

\begin{tabular}{|c|c|c|c|c|c|}
\hline & $C_{1}$ & $C_{2}$ & $C_{3}$ & $C_{4}$ & $C_{5}$ \\
\hline$A_{1}$ & $\left(g_{1.0}, g_{4.0}, g_{2.0}\right)$ & $\left(g_{3.0}, g_{4.0}, g_{3.0}\right)$ & $\left(g_{3.0}, g_{4.0}, g_{3.0}\right)$ & $\left(g_{3.0}, g_{7.0}, g_{4.0}\right)$ & $\left(g_{3.0}, g_{5.0}, g_{4.0}\right)$ \\
\hline$A_{2}$ & $\left(g_{1.0}, g_{4.0}, g_{1.0}\right)$ & $\left(g_{1.0}, g_{1.0}, g_{3.0}\right)$ & $\left(g_{3.0}, g_{2.0}, g_{1.0}\right)$ & $\left(g_{1.0}, g_{3.0}, g_{1.0}\right)$ & $\left(g_{4.0}, g_{2.0}, g_{0.0}\right)$ \\
\hline$A_{3}$ & $\left(g_{1.0}, g_{3.0}, g_{2.0}\right)$ & $\left(g_{3.0}, g_{0.0}, g_{4.0}\right)$ & $\left(g_{4.0}, g_{3.0}, g_{1.0}\right)$ & $\left(g_{4.0}, g_{3.0}, g_{2.0}\right)$ & $\left(g_{4.0}, g_{1.0}, g_{2.0}\right)$ \\
\hline$A_{4}$ & $\left(g_{2.0}, g_{3.0}, g_{1.0}\right)$ & $\left(g_{2.0}, g_{0.0}, g_{3.0}\right)$ & $\left(g_{4.0}, g_{2.0}, g_{1.0}\right)$ & $\left(g_{4.0}, g_{3.0}, g_{0.0}\right)$ & $\left(g_{4.0}, g_{0.0}, g_{0.0}\right)$ \\
\hline
\end{tabular}


Table 14

Linguistic neutrosophic decision matrix of Example 5.4.

\begin{tabular}{|c|c|c|c|c|c|}
\hline & $C_{1}$ & $C_{2}$ & $C_{3}$ & $C_{4}$ & $C_{5}$ \\
\hline$A_{1}$ & $\left(g_{1.0}, g_{4.0}, g_{1.0}\right)$ & $\left(g_{3.0}, g_{4.0}, g_{3.0}\right)$ & $\left(g_{3.0}, g_{4.0}, g_{3.0}\right)$ & $\left(g_{1.0}, g_{3.0}, g_{2.0}\right)$ & $\left(g_{3.0}, g_{5.0}, g_{4.0}\right)$ \\
\hline$A_{2}$ & $\left(g_{1.0}, g_{4.0}, g_{1.0}\right)$ & $\left(g_{2.0}, g_{0.0}, g_{3.0}\right)$ & $\left(g_{3.0}, g_{4.0}, g_{2.0}\right)$ & $\left(g_{3.0}, g_{7.0}, g_{4.0}\right)$ & $\left(g_{4.0}, g_{3.0}, g_{0.0}\right)$ \\
\hline$A_{3}$ & $\left(g_{2.0}, g_{4.0}, g_{2.0}\right)$ & $\left(g_{3.0}, g_{4.0}, g_{2.0}\right)$ & $\left(g_{4.0}, g_{3.0}, g_{1.0}\right)$ & $\left(g_{4.0}, g_{3.0}, g_{0.0}\right)$ & $\left(g_{3.0}, g_{4.0}, g_{3.0}\right)$ \\
\hline$A_{4}$ & $\left(g_{2.0}, g_{3.0}, g_{2.0}\right)$ & $\left(g_{1.0}, g_{2.0}, g_{3.0}\right)$ & $\left(g_{3.0}, g_{2.0}, g_{2.0}\right)$ & $\left(g_{3.0}, g_{4.0}, g_{3.0}\right)$ & $\left(g_{4.0}, g_{0.0}, g_{0.0}\right)$ \\
\hline
\end{tabular}

Table 15

The ranking results of different approaches

\begin{tabular}{lc}
\hline Approaches & Ranking orders \\
\hline Approach based on the bidirectional projection with $f=f_{1}\left(s_{i}\right)$ (Ye, 2017b) & $A_{4}>A_{1}>A_{3}>A_{2}$ \\
Approach based on the bidirectional projection with $f=f_{2}\left(s_{i}\right)$ (Ye, 2017b) & $A_{4}>A_{3}>A_{1}>A_{2}$ \\
Approach based on the symmetry measure with $f=f_{2}\left(s_{i}\right)$ (Tu et al., 2018) & $A_{4}>A_{1}>A_{3}>A_{2}$ \\
The proposed method based on the bidirectional projection & $A_{4}>A_{3}>A_{1}>A_{2}$ \\
\hline
\end{tabular}

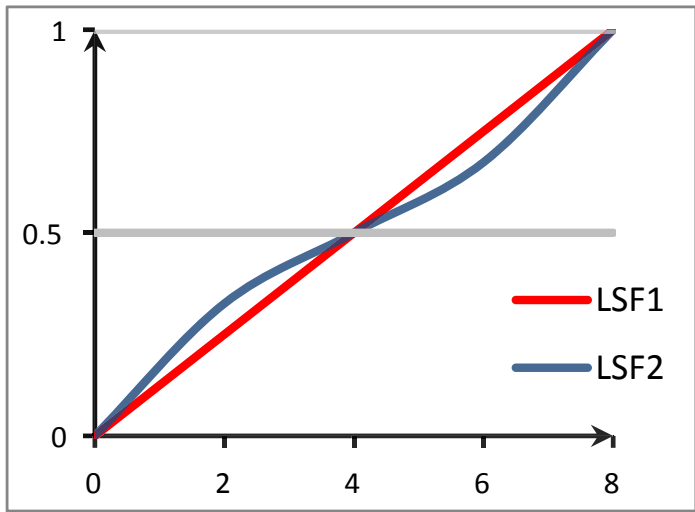

Fig. 1. Illustration of LSF1, LSF2.

From Table 15, it can be seen that the ranking result obtained by the proposed method is different from the ones by Tu et al.'s method (Tu et al., 2018). In the following, we give analysis about this experimental result as follows:

When comparing the proposed method with the Tu et al.'s method (Tu et al., 2018), there exist different evaluation information and measurement of two methods. Obviously, we have known linguistic evaluation can better describe the imprecise cognition and be closer to presentative judgement of human by comparing the Ye's MCDM method (Ye, 2017b) with our proposed method. Therefore, to further demonstrate the superiority of bidirectional projection, we compare the SVN-bidirectional projection (Ye, 2017b) with the SVN-weighted symmetry measure (Tu et al., 2018).

When comparing the Ye's MCDM method (Ye, 2017b) based on the SVN-bidirectional projection with Tu et al.'s method (Tu et al., 2018) based on SVN-weighted symmetry measure, we can see these two methods are based on the same evaluation information. The reason resulting in different ranking order of these two methods is because the different measurements are used to assess decision information. Specifically, the bidirectional projection measure use $B P_{r o j}\left(R^{*}, R_{i}\right)=\frac{1}{1+\left|\frac{R^{*} \cdot R_{i}}{\left\|R^{*}\right\|}-\frac{R^{*} \cdot R_{i}}{\left\|R_{i}\right\|}\right|}$ to obtain the bidirectional projection values between the ideal alternative $A^{*}$ and each alternative $A_{i}$, while the weighted symmetry measure is based on $M_{\varphi}\left(R^{*}, R_{i}\right)=\frac{1}{1+\left|\frac{R^{*} \cdot R_{i}}{\left\|R^{*}\right\|^{2}}-\frac{R^{*} \cdot R_{i}}{\left\|R_{i}\right\|^{2}}\right|}$ in this illustrated example. From the expression of $B P_{r o j}\left(R^{*}, R_{i}\right)$ and $M_{\varphi}\left(R^{*}, R_{i}\right)$, we can see there is a common advantage that they both consider the interactions between each alternative and the ideal alternative instead of only considering the projection/asymmetry measure of each alternative on the ideal alternative. The bidirectional projection measure is refined based on the projection measure, which considers the distance of each alternative and the closeness to the ideal alternative. The symmetry measure is proposed by improving an asymmetry measure that lacks of sufficient theoretical support. In a word, the proposed method is a new and effective MCDM model under linguistic neutrosophic environment.

Based on the comparisons and analysis above, the proposed method based on the bidirectional projection measure of LNNs has the advantage over the projection measure and the TOPSIS method in Liang et al. (2017) because it can consider the distance and included angle between options as well as the bidirectional projection magnitudes. Meanwhile, our method is more suitable in practical applications than the weighted symmetry measure of SVNs in Tu et al. (2018) and the bidirectional projection measure of SVNs in Ye (2017b) because LNNs can describe the uncertain and fuzzy evaluation information by LVs.

\section{Conclusion}

LNNs combine the advantages of LTs and SVNNs that they can express uncertain and incomplete information by LTs. Thus, they are a good tool to describe MCGDM evaluation information. In addition, the bidirectional projection measure can not only consider both the distance and the included angle but also consider the bidirectional projection between each alternative and the ideal solution. Based on the above, we develop the bidirectional projection-based MCGDM method with LNNs. What's more, we redefine a new distance of LNSs and build a weight determination model based on the fuzzy measure to obtain the objective weight vector of criteria, which considers the relationship between criteria and avoids the subjective preference or judgment of DMs. Finally, we verify the validity and show advantages of the proposed method by solving practical problem and comparing with other methods (Fang \& Ye, 2017; Liang et al., 2017; Tu et al., 2018; Ye, 2017b). The main contribution of this study is that the proposed method not only extended the bidirectional projection method to the field of LNNs, but also enriched the existing research theories of LNNs. In the future, we'd like to extend potential applications of the proposed method in different domains, such as supplier selection, transformer condition assessment, and product engineering and so on (Guan, Zhao, \& Du, 2017).

\section{Acknowledgment}

This paper is supported by the National Natural Science Foundation of China (Nos. 71771140, 71471172), the Special Funds of Taishan Scholars Project of Shandong Province (No. ts201511045), Shandong Provincial Social Science Planning Project (Nos. 17BGLJ04, 16CGLJ31 
and 16CKJJ27), the Natural Science Foundation of Shandong Province (No. ZR2017MG007).

\section{References}

Blagojevic, B., Srdjevic, B., Srdjevic, Z., \& Zoranovic, T. (2016). Heuristic aggregation of individual judgments in AHP group decision making using simulated annealing algorithm. Information Sciences, 330, 260-273.

Chen, Z. S., Chin, K. S., Li, Y. L., \& Yang, Y. (2016). Proportional hesitant fuzzy linguistic term set for multiple criteria group decision making. Information Sciences, 357, 61-87.

Cordon, O., \& Herrera, F. (2000). A proposal for improving the accuracy of linguistic modeling. IEEE Transactions on Fuzzy Systems, 8(3), 335-344.

Cui, W. H., Ye, J., \& Shi, L. L. (2018). Linguistic neutrosophic uncertain numbers and their multiple attribute group decision-making method. Journal of Intelligent \& Fuzzy Systems. https://doi.org/10.3233/JIFS-18331.

Dong, Y., Li, C. C., \& Herrera, F. (2016). Connecting the linguistic hierarchy and the numerical scale for the 2-tuple linguistic model and its use to deal with hesitant unbalanced linguistic information. Information Sciences, 367-368, 259-278.

Estrella, F. J., Espinilla, M., Herrera, F., \& Martínez, L. (2014). Flintstones: A fuzzy linguistic decision tools enhancement suite based on the 2-tuple linguistic model and extensions. Information Sciences, 280, 152-170.

Fan, C. X., Ye, J., Hu, K. L., \& Fan, E. (2017). Bonferroni mean operators of linguistic neutrosophic numbers and their multiple attribute group decision-making methods. Information, 8(3), 107.

Fang, Z. B., \& Ye, J. (2017). Multiple attribute group decision-making method based on linguistic neutrosophic numbers. Symmetry, 9, 111.

Grabisc, M., Nguyen, H. T., \& Walker, E. A. (1995). Fuzzy measures and integrals. Fundamentals of Uncertainty Calculi with Applications to Fuzzy Inference, 30, 107-171.

Guan, H., Zhao, A., \& Du, J. (2017). Enterprise green technology innovation behaviour. BeiJing: Economic Science Press.

Herrera, F., \& Martinez, L. (2000). A 2-tuple fuzzy linguistic representation model for computing with words. IEEE Transactions on Fuzzy Systems, 8(6), 746-752.

Liang, W. Z., Zhao, G. Y., \& Wu, H. (2017). Evaluating investment risks of metallic mines using an extended TOPSIS method with linguistic neutrosophic numbers. Sysmetry, 9(8), 149.

Liu, P. D., \& Guan, Z. L. (2009). Evaluation research on the quality of the railway passenger service based on the linguistic variables and the improved PROMETHEE-II method. Journal of Computers, 4(3), 265-270.

Liu, P. D., \& Wang, Y. M. (2014). Multiple attribute decision-making method based on single-valued neutrosophic normalized weighted Bonferroni mean. Neural Computing and Application, 25(7-8), 2001-2010.

Lourenzutti, R., \& Krohling, R. A. (2016). A generalized TOPSIS method for group decision making with heterogeneous information in a dynamic environment. Information Sciences, 330, 1-18.

Morente-Molinera, J. A., Kou, G., González-Crespo, R., Corchado, J. M., \& HerreraViedma, E. (2017). Solving multi-criteria group decision making problems under environments with a high number of alternatives using fuzzy ontologies and multigranular linguistic modelling methods. Knowledge-Based Systems, 137(1), 54-64.

Peng, H. G., \& Wang, J. Q. (2016). Hesitant uncertain linguistic Z-numbers and their application in multi-criteria group decision-making problems. International Journal of Fuzzy Systems, 19(5), 1300-1316.

Peng, J. J., Wang, J. Q., Wang, J., Yang, L. J., \& Chen, X. H. (2015). An extension of ELECTRE to multi-criteria decision-making problems with multi-hesitant fuzzy sets. Information Sciences, 307, 113-126.

Peng, J. J., Wang, J. Q., Zhang, H. Y., \& Chen, X. H. (2014). An outranking approach for multi-criteria decision-making problems with simplified neutrosophic sets.
Application Soft Computing, 25, 336-346.

Ren, Z. L., Xu, Z. S., \& Wang, H. (2017). Dual hesitant fuzzy VIKOR method for multicriteria group decision making based on fuzzy measure and new comparison method. Information Sciences, 388-389, 1-16.

Shapley, L. S. (1953). A value for n-person game. In H. Kuhn, \& A. Tucker (Eds.). Contributions to the theory of games. Princeton: Princeton University Press.

Shi, L. L., \& Ye, J. (2017). Cosine measures of linguistic neutrosophic numbers and their application in multiple attribute group decision-making. Information, 8(4), 117.

Simon, H. A. (1971). Administrative behavior-a study of decision making processes in administrative organization. New York: Macmillan Publishin Co, Inc.

Smarandache, F. (1999). A unifying field in logics. Neutrosophic: Neturosophic probability, set, and logic. Rehoboth: American Research Press.

Sugeno, M. (1974). Theory of fuzzy integral and its application. Doctorial dissertation, Tokyo Institute of Technology.

Tu, A. Y., Ye, J., \& Wang, B. (2018). Symmetry measures of simplified neutrosophic sets for multiple attribute decision-making problems. Symmetry, 10(5), 144.

Wang, H., Smarandache, F., Zhang, Y., \& Sunderraman, R. (2010). Single valued neutrosophic set. Mulispace Multistruct, 4, 410-413.

Wei, G. W. (2008). Maximizing deviation method for multiple attribute decision making in intuitionistic fuzzy setting. Knowledge-Based Systems, 21(8), 833-836.

Wei, G. W. (2010). GRA method for multiple attribute decision making with incomplete weight information in intuitionistic fuzzy setting. Knowledge-Based Systems, 23(3), 2443-3247.

Xu, Z. S. (2005). On method for uncertain multiple attribute decision making problems with uncertain multiplicative preference information on alternatives. Fuzzy Optimization and Decision Making, 4(2), 131-139.

Ye, J. (2014). Single valued neutrosophic cross-entropy for multicriteria decision making problems. Applied Mathematical Modelling, 38(3), 1170-1175.

Ye, J. (2015). An extended TOPSIS method for multiple attribute group decision making based on single valued neutrosophic linguistic numbers. Journal of Intelligent \& Fuzzy System, 28(1), 247-255.

Ye, J. (2017c). Bidirectional projection method for multiple attribute group decision making with neutrosophic numbers. Neural Computing and Applications, 28(5), 1021-1029.

Ye, J. (2017d). Simplified neutrosophic harmonic averaging projection-based method for multiple attribute decision making problems. International Journal of Machine Learning and Cybernetics, 8(3), 981-987.

Ye, J. (2017a). Linguistic neutrosophic cubic numbers and their multiple attribute decision-making method. Information, 8(3), 110.

Ye, J. (2017b). Projection and bidirectional projection measures of single-valued neutrosophic sets and their decision-making method for mechanical design schemes. Journal of Experimental \& theoretical artificial intelligence, 29(4), 731-740.

Ye, J. (2018a). Multiple attribute decision-making method based on linguistic cubic variables. Journal of Intelligent \& Fuzzy Systems, 34(4), 2351-2361.

Ye, J. (2018b). Multiple attribute decision-making methods based on expected value and similarity measure of hesitant neutrosophic linguistic numbers. Cognitive Computation, 10, 454-463.

Zadeh, L. A. (1965). Fuzzy sets, information. Control, 8(3), 338-353.

Zadeh, L. A. (1975). The concept of a linguistic variable and its application to approximate reasoning Part I. Information Sciences, 8(3), 199-249.

Zhang, X., \& Xu, Z. S. (2014). The TODIM analysis approach based on novel measured functions under hesitant fuzzy environment. Knowledge-Based Systems, 61(2), 48-58.

Zhang, Y., Xu, Z. S., \& Liao, H. (2017). A consensus process for group decision making with probabilistic linguistic preference relations. Information Sciences, 414, 260-275.

Zhou, W., \& Xu, Z. S. (2017). Group consistency and group decision making under uncertain probabilistic hesitant fuzzy preference environment. Information Sciences, 414(2), 276-288. 